\title{
Article \\ Multiphoton Bleaching of Red Fluorescent Proteins and the Ways to Reduce It
}

\author{
Mikhail Drobizhev ${ }^{1, *}$, Rosana S. Molina ${ }^{1}$ and Jacob Franklin ${ }^{2}$ \\ 1 Department of Microbiology and Cell Biology, Montana State University, Bozeman, MT 59717, USA; \\ zerlinia@gmail.com \\ 2 Vidrio Technologies LLC, 19955 Highland Vista Drive Suite 150, Ashburn, VA 20147, USA; \\ jacob@vidriotech.com \\ * Correspondence: mikhail.drobijev@montana.edu
}

Citation: Drobizhev, M.; Molina, R.S.; Franklin, J. Multiphoton Bleaching of Red Fluorescent Proteins and the Ways to Reduce It. Int. J. Mol. Sci. 2022, 23, 770. https://doi.org/ $10.3390 /$ ijms 23020770

Academic Editor: Kiryl D. Piatkevich

Received: 24 December 2021

Accepted: 7 January 2022

Published: 11 January 2022

Publisher's Note: MDPI stays neutral with regard to jurisdictional claims in published maps and institutional affiliations.

Copyright: (C) 2022 by the authors. Licensee MDPI, Basel, Switzerland. This article is an open access article distributed under the terms and conditions of the Creative Commons Attribution (CC BY) license (https:// creativecommons.org/licenses/by/ $4.0 /)$.
Abstract: Red fluorescent proteins and biosensors built upon them are potentially beneficial for twophoton laser microscopy (TPLM) because they can image deeper layers of tissue, compared to green fluorescent proteins. However, some publications report on their very fast photobleaching, especially upon excitation at $750-800 \mathrm{~nm}$. Here we study the multiphoton bleaching properties of mCherry, mPlum, tdTomato, and jREX-GECO1, measuring power dependences of photobleaching rates $K$ at different excitation wavelengths across the whole two-photon absorption spectrum. Although all these proteins contain the chromophore with the same chemical structure, the mechanisms of their multiphoton bleaching are different. The number of photons required to initiate a photochemical reaction varies, depending on wavelength and power, from 2 (all four proteins) to 3 (jREX-GECO1) to 4 (mCherry, mPlum, tdTomato), and even up to 8 (tdTomato). We found that at sufficiently low excitation power $P$, the rate $K$ often follows a quadratic power dependence, that turns into higher order dependence $\left(K \sim P^{\alpha}\right.$ with $\left.\alpha>2\right)$ when the power surpasses a particular threshold $P^{*}$. An optimum intensity for TPLM is close to the $P^{*}$, because it provides the highest signal-to-background ratio and any further reduction of laser intensity would not improve the fluorescence/bleaching rate ratio. Additionally, one should avoid using wavelengths shorter than a particular threshold to avoid fast bleaching due to multiphoton ionization.

Keywords: red fluorescent proteins; mCherry; mPlum; tdTomato; jREX-GECO1; multiphoton absorption; two-photon laser microscopy; multiphoton bleaching; photoionization; electron photodetachment

\section{Introduction}

Red fluorescent proteins (red FPs, RFPs) are genetically encoded molecular probes that find use in fluorescence microscopy of biological tissues, such as the brain [1-5]. Their red-shifted absorption and fluorescence provide structural and dynamic information from deeper layers of tissue, compared to their green counterparts, because red light penetrates better in scattering media. RFPs are particularly attractive for use in two-photon laser microscopy (TPLM) [6-9], where two near-infrared photons can effectively excite the RFP chromophore [10]. Two-photon excitation (2PE) spectra of RFPs fall in the range from 700 to $1200 \mathrm{~nm}[10,11]$ that matches the so-called tissue transparency window [12]. They show two main peaks in this range. The first one, at 1050-1200 nm, typically has moderate two-photon absorption (2PA) cross section, $\sigma_{2}=10-100 \mathrm{GM}\left(1 \mathrm{GM}=10^{-50} \mathrm{~cm}^{4} \mathrm{~s}\right)$. The second one, at 700-780 nm, is usually stronger, with $\sigma_{2}$ reaching 100-300 GM in some RFPs. This latter region corresponds to a higher energy, $S_{0} \rightarrow S_{m}$, transition that is also present in one-photon absorption spectra as a weak peak near 350-380 nm. When excited with two photons, it gains its intensity due to the nonlinear optical effect of pre-resonance enhancement that occurs when the laser photon energy approaches the lowest $S_{0} \rightarrow S_{1}$ transition energy from below, but still does not populate it $[13,14]$. Therefore, excitation at 
700-780 nm still corresponds to a simultaneous two-photon absorption with all advantages offered by TPLM, including high spatial (3D) resolution, low out-of-focus damage of tissue, and low autofluorescence background. Given the availability of commercial femtosecond Ti:sapphire lasers, with the high output power and stable operation in this spectral region and the possibility of dual-color two-photon imaging with simultaneous excitation of red and blue FPs [15], excitation at 700-780 nm provides a very attractive opportunity for TPLM with RFPs. However, researchers quickly discovered that RFPs bleach extremely fast when using femtosecond lasers operating in this wavelength range, with typical pulse-peak photon fluxes of $10^{27}-10^{29}$ photon $/ \mathrm{cm}^{2} / \mathrm{s}$ at the sample.

There are a number of reports investigating multiphoton bleaching of fluorescent dyes and FPs, but the molecular mechanisms remain almost completely unexplored. In their seminal work, Patterson and Piston reported on multiphoton bleaching of fluorescein dextran, aminocoumarin dextran, NADH, and Indo-1 [16]. Using a femtosecond $710 \mathrm{~nm}$ excitation, they found that although the initial fluorescence intensity increased quadratically with laser power $P$, the photobleaching rate $K$ increased much faster (i.e., according to a power law, $K \sim P^{\alpha}$ with the exponents $\alpha$ ranging from 3 to 5 ). This super-quadratic dependence suggested involvement of multiphoton processes with number of photons $n>2$. It is clear that after direct two-photon excitation, that requires very high photon flux, a large organic molecule can readily undergo several stepwise one-photon absorption events, through a ladder of electronic/vibrational excited states, that make a quasi-continuum above the $S_{1}$ level. The most general photochemical outcome of this process is multiphoton ionization or electron detachment (for anionic chromophores) [17]. For example, fluorescein dextran required absorption of a total of three photons (power exponent $3.1 \pm 0.1$ ) for photobleaching. Photodetachment energy of fluorescein lies between 5.1 and $6.3 \mathrm{eV}$ (depending on protonation state) [18]. For the $1.75 \mathrm{eV}$ photon energy (used in the Patterson and Piston experiment) the above numbers translate into the number of photons, $n \sim 3$, thus supporting the photodetachment mechanism. Photobleaching of aminocoumarin requires five photons (power exponent $5.1 \pm 0.2$ at $710 \mathrm{~nm}$ ) [16]. Given photoionization energy of aminocoumarin of $8.05 \mathrm{eV}$ [19], a minimum of five photons are required to ionize this molecule, which supports our assumption that the photobleaching is caused by multiphoton ionization.

Marchant and co-authors [20] demonstrated that the wild-type red FP, DsRed, bleaches in $\mathrm{CHO}$ cells according to a third power law $(\alpha=2.98 \pm 0.10)$ when excited at $760 \mathrm{~nm}$, in the range of average power densities $5-40 \mu \mathrm{W} / \mu \mathrm{m}^{2}$ (approximately corresponding to peak photon fluxes of $I_{0}=3 \times 10^{26}-2.5 \times 10^{27}$ photon $/ \mathrm{cm}^{2} / \mathrm{s}$ ). This suggests a three-photon initiated process. The authors also found that, upon excitation at $950 \mathrm{~nm}$, DsRed bleaches much slower than at $750 \mathrm{~nm}$ excitation, with the laser power adjusted to produce similar initial fluorescence signals at these two wavelengths. Later, Robinson and Marchant [21] extended this study to several DsRed mutants expressed in HEK-293 cells, and have shown that all of them bleach much faster at $750 \mathrm{~nm}$ compared to $950 \mathrm{~nm}$. The power exponents observed for bleaching at $750 \mathrm{mn}$ varied from 2.69 (for DsRed2) to 3.05 (for DsRed [N42Q]). A similar trend of slowing down the bleaching rate upon shifting excitation of DsRed2 to longer wavelengths, from 760 to 880 and further to $1100 \mathrm{~nm}$, was found in [22]. Higher than quadratic power dependence $(\alpha=2.9 \pm 0.10)$ was also observed in another DsRed mutant, tdRFP, expressed in T cells and excited at $1100 \mathrm{~nm}$ with $I_{0}=(2-3.5) \times 10^{29}$ photon $/ \mathrm{cm}^{2} / \mathrm{s}$ [23]. Interestingly, the same excitation fluxes resulted in much faster bleaching of enhanced green fluorescent protein (EGFP) at $850 \mathrm{~nm}$, with $\alpha=2.57 \pm 0.25$. Other groups also reported a super-quadratic dependence of photobleaching for EGFP and cyan FP (CFP) [24-27]. Kalies et al. correlated the power exponent of the EGFP bleaching rate with the multiphoton electron photodetachment from the chromophore or a nearby tryptophan residue [26].

Drobizhev and co-authors characterized multiphoton bleaching of monomeric RFPs, mFruits (mCherry, mPlum, and mStrawberry) and DsRed2 in buffer solution and E. coli cells, with the emphasis on possible photochemical mechanisms [28]. The rate of the first step of a chain of photochemical reactions leading to photobleaching was measured as a function of laser intensity at $790 \mathrm{~nm}$. The DsRed2 rate followed a power law with $\alpha=2.84 \pm 0.01$, at 
$I_{0}=(0.3-1.1) \times 10^{29} \mathrm{photon} / \mathrm{cm}^{2} / \mathrm{s}$, in agreement with previous observations [20,21]. In DsRed2, photobleaching occurs through the three-photon absorption process with a slight saturation of the one-photon $S_{m} \rightarrow S_{n}$ transition. Vertical detachment energy (VDE) of the anionic chromophore inside DsRed2 lies at $7.24 \mathrm{eV}$ [28], suggesting that at least five photons (of $790 \mathrm{~nm}$ ) are needed to reach this threshold. A three-photon-induced electron transfer reaction starting from the bound $S_{n}$ state of DsRed2 is probably a first step of phototransformation [28]. mFruits bleach according to a power law with $\alpha=3.2-3.5$, suggesting a four-photon mechanism with strong saturation of one or two one-photon transitions, following initial two-photon absorption step. Electron photodetachment energies of mFruits are found in the range 5.52 (mCherry) $-5.61 \mathrm{eV}$ (mPlum) [28]. Absorption of four photons (at $790 \mathrm{~nm}$ ) can easily provide enough energy to overcome this threshold and, therefore, the four-photon photodetachment is suggested as a first step of photobleaching in mFruits [28]. The chromophore environment of DsRed 2 contains more positive amino acid residues compared to mFruits, and this can be a reason of its higher photodetachment energy.

Since the fluorescence signal increases slower (quadratically), with the laser peak intensity compared to photobleaching rate, any reduction of the laser pulse-peak intensity should be beneficial until the moment where fluorescence signal becomes undetectable against the noise. Following this idea, several approaches to reduce photobleaching were suggested. Drummond et al. proposed to (1) block the laser with the programmed Pockels cell during the "flyback" time (when fluorescence is not collected but the laser still irradiates the sample); and (2) increase the sensitivity of detection by using an additional widefield detector in the transmission position, which made it possible to use less excitation power [25]. The use of passive pulse splitters, providing less peak intensity but higher repetition rate, resulted in faster reduction of photobleaching relatively to fluorescence signal [29]. On the other hand, if multiphoton absorption involves triplet manifold, lower pulse repetition rate can be beneficial [30]. Phase modulation or dispersion compensation of femtosecond pulses also helped reducing multiphoton bleaching [27,31-33]. Although these methods provide some improvement on the fluorescence/bleaching ratio, they require technical upgrades of commercial $2 \mathrm{P}$ microscopes.

Here we aim at finding simple solutions to the problem of rapid multiphoton bleaching in terms of optimization of laser intensity and excitation wavelength. They require the (phenomenological) knowledge of photochemical mechanisms and their dependence on laser intensity and wavelength. First, one can expect that at small laser intensities the bleaching rate should show a quadratic dependence on intensity, with the rate equal to that observed with one-photon excitation (with the $2 \mathrm{P}$ and $1 \mathrm{P}$ excitation rates being equal). A transition between quadratic and higher-order dependence defines a particular threshold for laser power, $P^{*}$. An optimum intensity for TPLM should be chosen close to the $P^{*}$ value because it provides the highest signal-to-background ratio (SBR), and any further reduction of intensity would not improve the fluorescence/bleaching ratio. A particular value of threshold intensity is a function of wavelength because the corresponding laser and molecular parameters depend on wavelength. Second, if the bleaching is due to multiphoton ionization (electron detachment) with $n$ photons involved (like in mFruits), one can look for a threshold photon energy (wavelength) where this process in not allowed energetically: $n h v<V D E$.

We check these ideas for a series of RFPs, including popular monomeric variants mCherry [1] and mPlum [34], one of the two-photon brightest RFP, tdTomato [1,10], and a new red $\mathrm{Ca}^{2+}$ sensor with the long Stokes shift in the $\mathrm{Ca}^{2+}$-bound state, jREX-GECO1 [35]. Compared to the previous work [28], we study photobleaching rates in a broader range of excitation intensities and at different excitation wavelengths across the 2PE spectrum. We found some important threshold values for the laser photon fluxes and wavelengths for these RFPs and show that in some cases one can predict these values if the molecular and laser parameters are known. 


\section{Results}

\subsection{Physical Model of Multiphoton Bleaching in a Thin Colony of Cells Containing} Fluorescing Molecules

We use a physical model of multiphoton bleaching that was previously described in [28]. Here we briefly present its main points and summarize its results.

After absorption of $n$ photons, a molecule can undergo a chain of chemical transformations leading to eventual bleaching of fluorophore,

$$
A^{*} \rightleftarrows B^{(*)} \rightarrow C \rightarrow
$$

where $A$ is the concentrations of the initial form, and $B, C$, etc. are the concentrations of intermediate forms. The asterisk denotes an electronically excited state. This process results in a complex, non-monoexponential decay kinetics of the initial state $A$. For simplicity, here we measure the initial slope of the fluorescence decay curve $F(t)$ normalized to 1 at $t=0$ (method of initial rates of chemical reaction). This slope provides the observable rate $K$ that, in turn, is related to molecular rate of the first reaction step, $k_{1}$, corresponding to the $A^{*} \rightarrow B^{(*)}$ transition. To express $k_{1}$ through molecular and laser parameters, we consider dynamics of populations in several different energy level diagrams displayed in Figure 1.

(a)

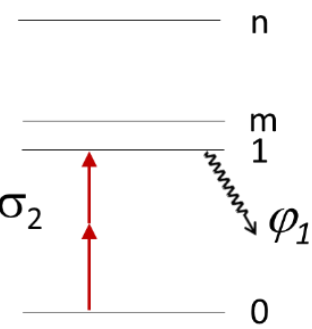

(c)

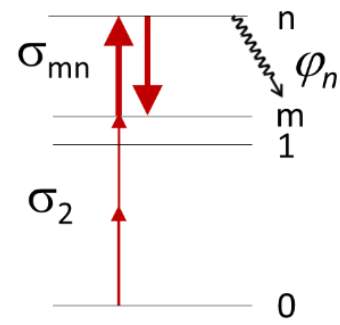

(b)

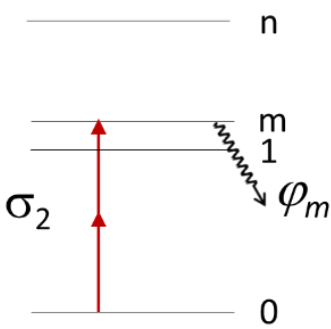

(d)

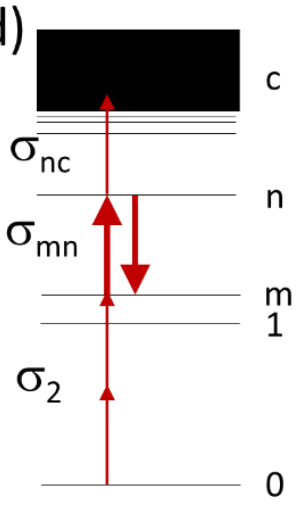

Figure 1. Energy level diagrams for some multiphoton induced photobleaching processes. Panels $(\mathbf{a}, \mathbf{b})$ correspond to two-photon bleaching following initial two-photon absorption with the cross section $\sigma_{2}$. Panel (c) corresponds to a three-photon bleaching where initial 2P excitation to level $\mathrm{m}$ ( $\mathrm{m}$ can be equal to 1 ) is followed by a one-photon transition $\mathrm{m} \rightarrow \mathrm{n}$ with the 1PA cross section, $\sigma_{\mathrm{mn}}$. The bold arrows connecting $\mathrm{m}$ and $\mathrm{n}$ states designate strong absorption (up) and stimulated emission (down) resulting in possible saturation of the $\mathrm{m} \rightarrow \mathrm{n}$ transition. Panel (d) shows a four-photon bleaching process, where, after three-photon excitation to level $n$, an electron leaves a molecule with photoionization (photodetachment) cross section $\sigma_{\mathrm{nc}}$, and transits into a continuum of states $(\mathbf{c})$. 
The diagram (a) represents a low intensity (lower than a certain threshold) two-photon excitation of molecule to the first excited electronic level $S_{1}$, from which it can start a transformation with quantum yield $\varphi_{1}$. The rate $k_{1}$ depends quadratically on laser power in this regime. This process should obey the well-known Kasha's rule that states that independently of the mode of excitation (i.e., one- or two-photon), the quantum yield $\varphi_{1}$ should be the same.

The diagram (b) corresponds to a low-intensity two-photon excitation to a higher electronic level $S_{m}$. After such excitation, a molecule can either relax to the $S_{1}$ state with subsequent transformation to $B$ as in case (1) (standard Kasha's pathway), or proceed to $B$ with different quantum yield, $\varphi_{\mathrm{m}}$ (violating Kasha's rule). The latter corresponds to a situation when the $A^{*} \rightarrow B^{(*)}$ transition efficiency depends on the excess excitation energy in the $A^{*}$ state relatively to the lowest level of the $S_{1}$ state. One can think that this can only happen if the photochemical quantum yield $\varphi_{\mathrm{m}} \sim 1$, because the photochemical rate should be on the picosecond scale (i.e., competing with intramolecular vibrational relaxation). However, the possibility of the reverse reaction $B^{*} \rightarrow A^{*}$ or even $B^{*} \rightarrow B \rightarrow A$ removes that stringent requirement and the $\varphi_{\mathrm{m}}$ value is still allowed to be much less than 1 . In other words, a violation of Kasha's rule is related to a presence of a second specie, $B^{*}$ that is dynamically coupled to $A^{*}$.

The third diagram (c) represents the three-photon process where the initial simultaneous two-photon excitation $(0 \rightarrow \mathrm{m})$ is followed by a third photon, absorbed linearly through the $\mathrm{m} \rightarrow \mathrm{n}$ transition. The quantum yield of the reaction, initiated from the excited state $\mathrm{n}, \varphi_{\mathrm{n}}$, can be different from $\varphi_{1}$ and from $\varphi_{\mathrm{m}}$, and the same considerations as applied to $\varphi_{\mathrm{m}}$ can be used here. This three-photon $(2+1)$-induced process occurs at moderate laser intensities, typical for TPLM. Even with moderately high intensities needed for the robust two-photon excitation $\left(10^{28}-10^{29}\right.$ photons $\left./ \mathrm{sm}^{2} / \mathrm{s}\right)$, once a molecule is in the $\mathrm{m}$ state, the probability of the $\mathrm{m} \rightarrow \mathrm{n}$ transition (bold up arrow in Figure 1c) can be very high. This will result in partial saturation of transition. In fact, for typical one-photon cross sections, $\sigma_{\mathrm{mn}} \sim 10^{-16} \mathrm{~cm}^{2}$, the probability of absorption during the pulse duration $\left(10^{-13} \mathrm{~s}\right)$ becomes $\sim 0.1-1$, and the stimulated emission must be considered as well (bold down arrow in Figure 1c). As a result, the intensity dependence of the rate will show a power dependence with an exponent $\alpha$ between 2 and 3. The last diagram (d) demonstrates the process of four-photon $(2+1+1)$-induced bleaching involving initial two-photon absorption followed by sequential one-photon absorptions of two additional photons, eventually bringing the electron to an ionization continuum that lies above the ionization energy (electron detachment energy for anionic chromophores). The last step transition $\mathrm{n} \rightarrow \mathrm{c}$ typically has small cross sections, $\sigma_{\mathrm{nc}} \sim 10^{-20}-10^{-19} \mathrm{~cm}^{2}$ ([17] and the data below).

Our estimation shows that with the intensities used here, it is difficult to saturate this transition. In the case of partial saturation of the $m \rightarrow n$ transition, the power exponent of the bleaching rate will be between 3 and 4 .

The solution of the rate equations corresponding to cases (a)-(d) at a particular photon flux for "local" decay rates was given in [28]. In our experiment, we irradiate E. coli cells expressing a particular RFP in a 2PLM setup and measure the temporal decay of fluorescence signal. In the description of the observed multiphoton bleaching kinetics, we neglect the diffusion of FPs in and out of laser focal area during bleaching time because the laser beam diameter in our experiment is much larger than the E. coli cell size. However, because of the spatial distribution of intensity, different chromophore molecules will see different photon fluxes. To obtain the observed (average) rates we had to integrate the local rates over space and time. It is important that the colony thickness $l$ in our experiments is comparable to the Rayleigh length $z_{R}$ of the laser intensity distribution. Therefore, we do not use a "thick sample" approximation and the integration results depend on geometrical parameter equal to the ratio $\eta=l / 2 z_{R}$. 
To proceed with integration, we describe the spatial and temporal distribution of laser intensity near the focal spot by a Gaussian-Lorentzian function in cylindrical coordinates,

$$
\begin{aligned}
& I(r, z, \tau)=I_{0} \frac{w_{0}^{2}}{w(z)^{2}} \exp \left(-\frac{2 r^{2}}{w(z)^{2}}\right),-\Delta \tau / 2 \leq \tau<\Delta \tau / 2 \\
& I(r, z, \tau)=0, \text { otherwise, }
\end{aligned}
$$

where $w(z)$ is the beam waist radius at distance $z$ from the focal plane (in $\left.\mathrm{cm}^{2}\right), \tau$ is the time lapse within the pulse (in s), $I_{0}=I(0,0,0)$ is the peak photon density (in photons $/ \mathrm{cm}^{2} \mathrm{~s}$ ) in focal spot. For simplicity, we consider the laser pulse temporal profile as rectangular with a full width of $\Delta \tau$. Integration of (2) over time and cross-sectional area at focal plane $z=0$ $\left(w=w_{0}\right)$ relates the peak photon density $I_{0}$ to the average laser power $P($ in $W)$, measured in experiment:

$$
P=\frac{\pi}{2} f h v \Delta \tau w_{0}^{2} I_{0}
$$

where $f$ is the pulse repetition rate, $h$ is the Planck constant, $v$ is the photon frequency (in $\mathrm{Hz}$ ).

We now calculate the initial decay rate of the observed fluorescence signal normalized to initial signal $(F(0))$ as follows:

$$
K=-\left.\frac{1}{F(0)} \frac{d F}{d t}\right|_{t=0}=-\frac{\left.\int d V \int_{p u l s e} d \tau I^{2}(r, z, t) \frac{\partial n(r, z, t)}{\partial t}\right|_{t=0}}{n(0) \int d V \int_{p u l s e} d \tau I^{2}(r, z, t)}
$$

where $t$ is the measurement time, $d V$ is the element of volume $d V=2 \pi r d r d z, n(r, z, t)$ is the time-dependent local concentration of unbleached molecules.

In general, the multiphoton rate can contain different terms corresponding to processes with different orders that potentially compete with each other:

$$
K=K^{(2)}+K^{(3)}+K^{(4)}+\ldots
$$

We now provide the analytical results, corresponding to individual terms in the above equation without and with partial saturation of some of the intermediate transitions. In the case (a) of Figure 1, the observed rate of two-photon bleaching process reads

$$
K^{(2)}=\frac{1}{\pi^{2}} \sigma_{2} \varphi_{1} \frac{\Gamma_{3}(\eta)}{\Delta \tau f(h v)^{2} w_{0}^{4}} P^{2}
$$

where all $\Gamma_{\mathrm{i}}(\eta)$ functions, $\mathrm{i}=2,3,4,5$, appearing here and throughout depend only on the ratio $\eta=l / 2 z_{R}$. They are presented in Supplementary Info. In the case (b) of Figure 1, one can use the same expression for $K^{(2)}$ where $\varphi_{1}$ is replaced by $\varphi_{\mathrm{m}}$.

In the case of three-photon initiated process (c) without saturation one obtains,

$$
K^{(3)}=\frac{4}{5 \pi^{3}} \sigma_{2} \sigma_{\operatorname{mn}} \varphi_{\mathrm{n}} \frac{\Gamma_{4}(\eta)}{\Delta \tau f^{2}(h v)^{3} w_{0}^{6}} P^{3}
$$

In the case of slight saturation of the $\mathrm{m} \rightarrow \mathrm{n}$ transition (i.e., when the observed power exponent is slightly less than 3), one can use the following fitting function:

$$
K^{(3)}=\frac{1}{\pi^{2}} \frac{\sigma_{2} \varphi_{n}}{\Delta \tau f(h v)^{2} w_{0}^{4}}\left[\frac{1}{5} \Gamma_{4}(\eta) \frac{P}{P_{S}}-\frac{1}{18} \Gamma_{5}(\eta)\left(\frac{P}{P_{S}}\right)^{2}\right] P^{2}
$$

where

$$
P_{S}=\frac{\pi}{4} \frac{f h v w_{0}^{2}}{\sigma_{\mathrm{mn}}}
$$

is the saturation power (in W). 
In the case of four-photon initiated process without saturation, Figure 1c, the observed rate reads

$$
K^{(4)}=\frac{4}{9 \pi^{4}} \sigma_{2} \sigma_{\mathrm{mn}} \sigma_{\mathrm{nc}} \frac{\Gamma_{5}(\eta)}{\Delta \tau f^{3}(h v)^{4} w_{0}^{8}} P^{4}
$$

For the strong saturation of the $\mathrm{m} \rightarrow \mathrm{n}$ transition, (i.e., when the observed power exponent is slightly larger than 3), one can use an approximation:

$$
K^{(4)}=\frac{1}{4 \pi} \frac{\sigma_{2} \sigma_{\mathrm{nc}}}{\sigma_{\mathrm{mn}}^{2}} \frac{1}{\Delta \tau f(h v)^{2} w_{0}^{4}}\left[\frac{1}{10} \Gamma_{4}(\eta)\left(\frac{P}{P_{S}}\right)^{2}-\frac{1}{4} \Gamma_{3}(\eta)\left(\frac{P}{P_{S}}\right)+\frac{1}{3} \Gamma_{2}(\eta)\right] P .
$$

\subsection{Multiphoton Bleaching of mCherry in E. coli Colonies}

We first measure the relative corrected 2PE spectrum of mCherry in E. coli cells from the same colony that we use in bleaching experiments and compare it to the spectrum previously observed in buffer solution [11]. Both spectra are shown in Figure 2 (top), with the spectrum in E. coli normalized to the 2PA cross section of mCherry in solution at $760 \mathrm{~nm}$. The two spectra overlap quite well, confirming that the E. coli colony does express mCherry. The first electronic transition $\left(\mathrm{S}_{0} \rightarrow \mathrm{S}_{1}\right)$ occurs at 1000-1200 nm, and the higher-energy and stronger electronic transition $\left(\mathrm{S}_{0} \rightarrow \mathrm{S}_{\mathrm{m}}\right)$ emerges at wavelengths shorter than $800 \mathrm{~nm}$.
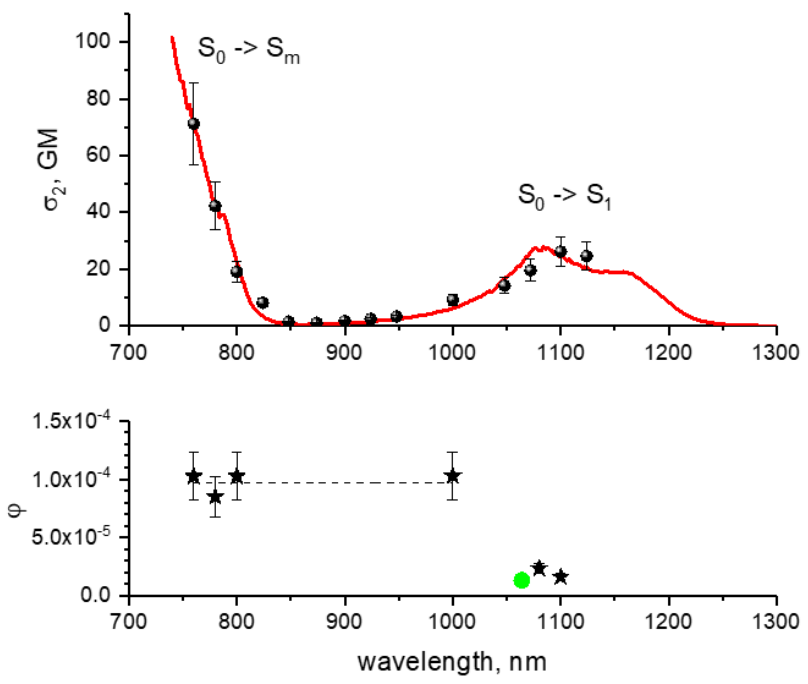

Figure 2. Top: Two-photon absorption spectrum of mCherry in purified buffer solution at $\mathrm{pH} 8$, (red line) [11] and two-photon excitation spectrum of mCherry measured in E. coli cells (symbols) (normalized to the 2PA cross section of solution spectrum at $760 \mathrm{~nm}$ ). Bottom: Quantum yield of the first step of photobleaching process initiated by absorption of two photons as a function of excitation wavelength (black asterisks) for mCherry in E. coli. Quantum yield of the first step of one-photon induced photobleaching process upon laser excitation at $532 \mathrm{~nm}$ [36] is shown by a green circle at double wavelength $(1064 \mathrm{~nm})$.

Next, we measured the power dependences of the bleaching rates of mCherry expressed in $E$. coli cells in a broad range of powers and at several wavelengths across the 2PA spectrum. The bleaching wavelengths correspond to the positions of asterisks in the bottom panel of Figure 2. They fall in both the $S_{0} \rightarrow S_{1}$ and $S_{0} \rightarrow S_{m}$ absorption bands. Figure 3 shows an example of the six fluorescence decay curves obtained at $800 \mathrm{~nm}$ upon bleaching at six different points within one colony with different laser powers $P$. For two of them, corresponding to $P=37$ and $70 \mathrm{~mW}$, we show here the linear fits of the initial decay stage (dashed lines). The $K$ values were determined as the negative slopes of these lines (cf. Equation (4)). A very sharp increase of the decay rate is observed with the increase of intensity. 


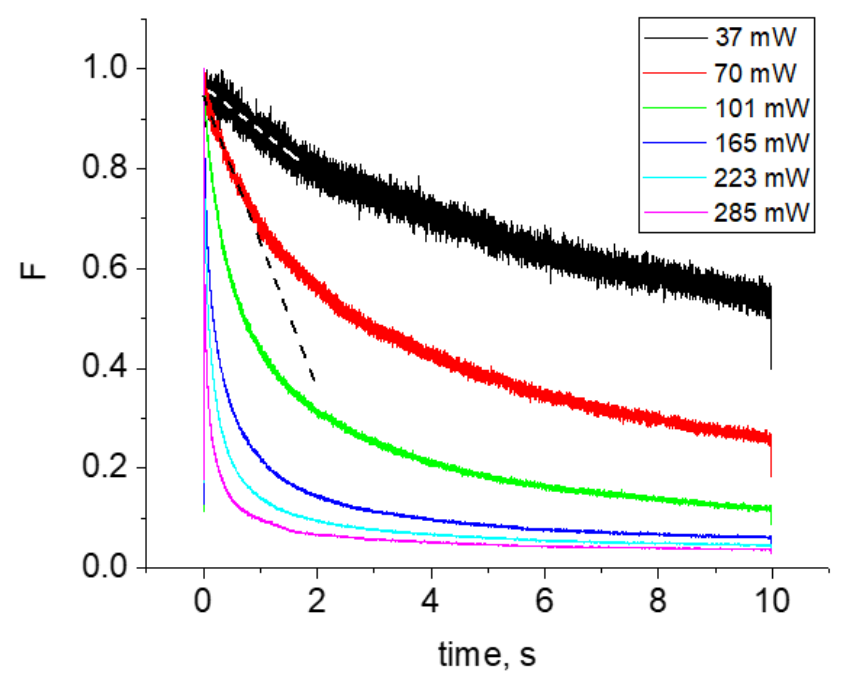

Figure 3. Fluorescence decay kinetic curves obtained at $\lambda=800 \mathrm{~nm}$ at different power values at the sample, shown in the inset. The kinetics are normalized to the $F(0)=1$ values. The dashed lines illustrate the derivatives of the decay curves at $t=0$, corresponding to 37 and $70 \mathrm{~mW}$.

Figure 4 shows the series of power dependences of the rate $\mathrm{K}$ obtained at different excitation wavelengths. We observe several interesting trends.

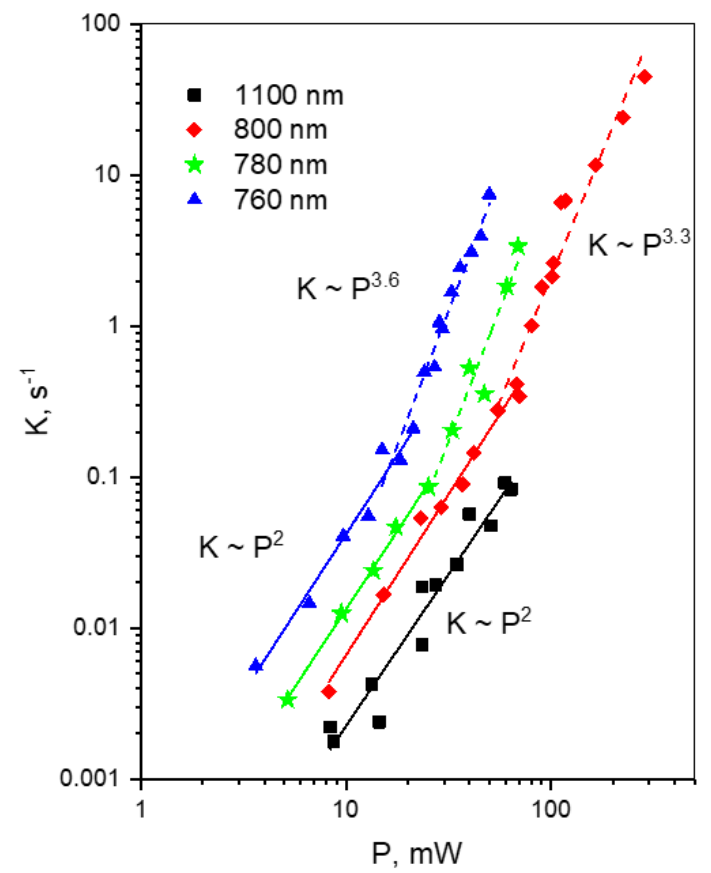

Figure 4. Power dependences of the initial bleaching rate of mCherry at different excitation wavelengths (see legend in the inset), presented in double logarithmic scale.

(1) For each wavelength, there is a range of power values where the bleaching rate depends quadratically on power. Although this behavior is natural and reflects the fundamental bleaching limit corresponding to photochemical reaction starting just after absorption of two photons, to the best of our knowledge there are no publications where quadratic law was shown for any FP. After reaching a certain threshold, the dependence switches to super-quadratic, with $\alpha=3.3-3.6$. Note that previously the bleaching of mCherry was measured at $790 \mathrm{~nm}$ at similar excitation conditions (same objective lens, similar colony thickness and pulse duration) with the laser power of 40-150 $\mathrm{mW}$ [28], and the $K \sim P^{3.4}$ dependence was observed. As one can see from Figure 4 , these power levels 
were higher than the threshold (at 780-800 $\mathrm{nm}$ ) and therefore the quadratic part was missed there.

(2) The power threshold $P^{*}$ for the super-quadratic transition is observed for wavelengths $760-800 \mathrm{~nm}$, and it shifts to lower power values as the wavelengths becomes shorter.

(3) We did not observe a transition from quadratic to super quadratic dependence for wavelengths of 1100 (black squares) and $1000 \mathrm{~nm}$ (data not shown), at least for highest available power levels. Given the vertical electron detachment energy of mCherry is $5.52 \mathrm{eV}$ [28], one can predict that the absorption of four photons with energy less than $1.57 \mathrm{eV}(>900 \mathrm{~nm})$ would not suffice to photodetach an electron. As a result, we would not anticipate the transition from $P^{2}$ to $P^{3.5}$ dependence for wavelengths longer than $900 \mathrm{~nm}$. This imposes a critical wavelength for two-photon excitation of mCherry, $\lambda=900 \mathrm{~nm}$, suggesting that using the lowest, $S_{0} \rightarrow S_{1}$ transition at 1000-1100 nm should result in slower (quadratic) multiphoton bleaching even at high powers.

To analyze the quadratic segments of the power dependences at different wavelengths (Figure 4), we plot the data obtained in the low power regime in the $K$ vs. $P^{2}$ coordinates and fit them to the straight line to obtain the slope that, according to (5), is

$$
a_{2}=\frac{1}{\pi^{2}} \sigma_{2} \varphi_{1} \frac{\Gamma_{3}(\eta)}{\Delta \tau f(h v)^{2} w_{0}^{4}}
$$

(Supplementary Figures S2-S6). Knowing all of the laser parameters (Methods) and the 2PA cross sections for mCherry [11], we calculate the photochemical quantum yields $\varphi_{1, \mathrm{~m}}$ from the factor $a_{2}$, see Table 1 and Figure 2, bottom.

Table 1. Molecular photobleaching parameters of mCherry at different wavelengths.

\begin{tabular}{|c|c|c|c|c|c|c|}
\hline$\lambda, \mathrm{nm}$ & $\mathbf{n}$ & $\sigma_{2}, \mathrm{GM}^{(\mathrm{a})}$ & $\varphi_{1, \mathrm{~m}}{ }^{(\mathrm{b})}$ & $\sigma_{\mathrm{mn}} \sigma_{\mathrm{nc}}, \mathrm{cm}^{4(\mathrm{c})}$ & $\sigma_{\mathrm{mn}}, \mathrm{cm}^{2(\mathrm{~d})}$ & $\sigma_{\mathrm{nc}}, \mathrm{cm}^{2(\mathrm{~d})}$ \\
\hline 1100 & 2 & 24 & $(1.6 \pm 0.3) \times 10^{-5}$ & - & - & - \\
\hline 1000 & 2 & 6.2 & $(1.0 \pm 0.2) \times 10^{-4}$ & - & - & - \\
\hline 800 & 2 to 4 & 22 & $(1.0 \pm 0.2) \times 10^{-4}$ & $(5.0 \pm 0.8) \times 10^{-36}$ & $(3.7 \pm 1.4) \times 10^{-17}$ & $(1.3 \pm 0.5) \times 10^{-19 *}$ \\
\hline 780 & 2 to 4 & 42 & $(8.5 \pm 1.7) \times 10^{-5}$ & $(1.7 \pm 0.3) \times 10^{-35}$ & $(5.0 \pm 1.6) \times 10^{-17}$ & $(3.4 \pm 0.9) \times 10^{-19}$ \\
\hline 760 & 2 to 4 & 71 & $(1.0 \pm 0.2) \times 10^{-4}$ & $(3.3 \pm 0.5) \times 10^{-35}$ & $(8.8 \pm 1.9) \times 10^{-17}$ & $(7.3 \pm 1.2) \times 10^{-19}$ \\
\hline
\end{tabular}

Column 2 shows the number of photons $n$ involved in the process. (a) 2PA cross sections are taken from [11] (b) Found from the fits of $K(P)$ dependences to Function (5) in the low power range below the threshold. (c) Found from the fits of $K(P)$ dependences to Function (9) in the power range just above the threshold. (d) Found from the fits of $K(P)$ dependences to Function (10) in the power range above the threshold. * Obtained by dividing $\sigma_{\mathrm{mn}} \sigma_{\mathrm{nc}}$ (column 5) by $\sigma_{\mathrm{mn}}$ (column 6).

Interestingly, upon excitation at $1100 \mathrm{~nm}$, the photochemical quantum yield, $\varphi_{1}=(1.6 \pm 0.3) \times 10^{-5}$, is within experimental error equal to the value previously obtained for mCherry in buffer solution using one-photon excitation (at $532 \mathrm{~nm}$ ), $\varphi_{1}=1.3 \times 10^{-5}$ [36]. Several important conclusions stem from this result: (1) Our physical model of multiphoton bleaching in thin films catches correctly the main factors. (2) Multiphoton bleaching rate (at least at low intensities) does not depend on local environment of an FP molecule, i.e., buffer vs. E. coli cell (cf. [28]). (3) The Kasha's rule holds at long-wavelength excitation (i.e., the photochemical rate is the same for the excitation accomplished either with one or two photons).

However, in the shorter wavelengths region, $760-1000 \mathrm{~nm}$, the quantum yield $\varphi_{\mathrm{m}}$ is $\sim$ five to six-fold larger, but does not depend on wavelength. Such violation of Kasha's rule can be due to lowering of a potential barrier for photochemical reaction when the chromophore gains larger electronic/vibrational energy in the excited state, as discussed in Section 2.1, see Figure 1b. Similar effect was observed for DsRed protein in [37].

With the increasing laser intensity, the dependences in Figure 4 measured at 760, 780 , and $800 \mathrm{~nm}$ start to grow faster than quadratic, with the power exponent between 
3 and 4 . When we subtract the $K^{(2)}=a_{2} P^{2}$ term (with the $a_{2}$ found above) from the observed $K(P)$ dependence, we find that in a certain power range, where $P$ is higher than the threshold, $P^{*}$, but still is not saturated, the dependence is well described by a 4-th power law: $K^{(4)}=K-a_{2} P^{2}=a_{4} P^{4}$. The plot of $K^{(4)}$ vs. $P^{4}$ (Supplementary Figures S7-S9) presents a straight line with the slope $a_{4}$ that, according to Equation (9), reads

$$
a_{4}=\frac{4}{9 \pi^{4}} \sigma_{2} \sigma_{\mathrm{mn}} \sigma_{\mathrm{nc}} \frac{\Gamma_{5}(\eta)}{\Delta \tau f^{3}(h v)^{4} w_{0}^{8}}
$$

After substitution of all the laser and geometric parameters, we find a product of two cross sections, $\sigma_{\mathrm{mn}} \sigma_{\mathrm{nc}}$, corresponding to two consecutive steps, see Table 1 . This value strongly depends on wavelength, increasing by an order of magnitude upon tuning the laser from 800 to $760 \mathrm{~nm}$.

Once the molecular parameters $\sigma_{\mathrm{mn}} \sigma_{\mathrm{nc}}$ and $\varphi_{\mathrm{m}}$ are known, one can predict the $P^{*}$ value if the geometry of the sample illumination and laser parameters (beam waist at the sample, pulse repetition rate, and wavelength) are known. These estimations can provide useful guidelines for researchers aiming to avoid super-quadratic photobleaching. For example, if the multiphoton process at $P>P^{*}$ involves four-photon induced reaction, then equating right hand sides of Equations (5) and (9) gives:

$$
P^{*}=\frac{3 \pi}{2} \sqrt{\frac{\varphi_{\mathrm{m}}}{\sigma_{\mathrm{mn}} \sigma_{\mathrm{nc}}}} \sqrt{\frac{\Gamma_{3}}{\Gamma_{5}}} f h v w_{0}^{2} .
$$

Using Equation (13), we calculated the predicted threshold power for mCherry for our specific experimental conditions. The results are shown in Table 2 together the with $P^{*}$ values estimated from the "bending" points of the power dependences of Figure 4. The predicted and observed $P^{*}$ values correlate well. This validates our estimation of the laser and sample parameters $w_{0}$ and $\Gamma_{3} / \Gamma_{5}$ entering Equation (13). As one can see, the main factor responsible for the reduction of $P^{*}$ at shorter wavelength is the increase of the $\sigma_{\mathrm{mn}} \sigma_{\mathrm{nc}}$ value.

Table 2. Threshold laser powers $P^{*}$ and peak photon flux $I_{0}^{*}$ for the transition from quadratic to

\begin{tabular}{|c|c|c|c|c|}
\hline$\lambda, \mathrm{nm}$ & $\begin{array}{c}\left(\varphi_{\mathrm{m}} / \sigma_{\mathrm{mn}} \sigma_{\mathrm{nc}}\right)^{1 / 2} \\
\mathrm{~cm}^{-2}\end{array}$ & $w_{0}^{2}, \mathrm{~cm}^{2}$ & $\begin{array}{c}P^{*} \text { Predicted, } \mathrm{mW} \\
\left(I_{0}{ }^{*} \text {, photons } / \mathrm{cm}^{2} / \mathrm{s}\right)\end{array}$ & $\begin{array}{c}P^{*} \text { Observed, } \mathrm{mW} \\
\left(I_{0}{ }^{*}, \text { photons } / \mathrm{cm}^{2} / \mathrm{s}\right)\end{array}$ \\
\hline 760 & $1.7 \times 10^{15}$ & $1.32 \times 10^{-7}$ & $26\left(3.6 \times 10^{28}\right)$ & $19\left(2.6 \times 10^{28}\right)$ \\
\hline 780 & $2.2 \times 10^{15}$ & $1.37 \times 10^{-7}$ & $34\left(3.9 \times 10^{28}\right)$ & $28\left(3.2 \times 10^{28}\right)$ \\
\hline 800 & $4.5 \times 10^{15}$ & $1.43 \times 10^{-7}$ & $68\left(5.4 \times 10^{28}\right)$ & $61\left(4.8 \times 10^{28}\right)$ \\
\hline
\end{tabular}
super-quadratic dependence of the photobleaching rate for mCherry.

Column 2 presents a combination of molecular parameters that define the $P^{*}$ value. Column 3 shows the laser waist squared that is also responsible for $P^{*}$.

At even higher laser powers, the $K(P)$ starts to saturate (i.e., becomes slower than the 4-th power). Fitting the power dependence in this region with the model Function (10) (Supplementary Figure S10) makes it possible to find individual values of the $\sigma_{\mathrm{mn}}$ and $\sigma_{\mathrm{nc}}$ cross sections, see Table 1.

Knowing these values, we can now compare the bleaching rates of mCherry in two very different experimental settings. (1) A high repetition rate $(80 \mathrm{MHz})$ laser with relatively low pulse energy is focused to a few microns spot onto a thin film of live E. coli colony expressing mCherry; and (2) a low repetition rate $(1 \mathrm{kHz})$ regenerative amplifier laser beam with very high pulse energy and uniform power density across the $3 \mathrm{~mm}$ diameter passes through a buffer solution of purified mCherry. For the case (2), mCherry bleaches at an initial rate of $k_{1}=2.8 \times 10^{-3} \mathrm{~s}^{-1}$ when the laser intensity is $11.4 \mathrm{~W} / \mathrm{cm}^{2}$ [28]. It is interesting to check if, by taking the molecular parameters obtained here for bleaching in setting (1), we still would be able to reproduce the rate found in solution with an amplifier setup. The 
rate $k_{1}$ for the homogeneous distribution of power (i.e., without spatial averaging), but with possible saturation (Figure 1d), reads [28]:

$$
k_{1}^{(4)}=\frac{1}{32} \frac{\sigma_{2} \sigma_{\mathrm{nc}}}{\sigma_{\mathrm{mn}}^{3}} \frac{f}{\Delta \tau}\left[\frac{1}{2} X^{3}-X^{2}+X-X e^{-X}\right],
$$

where $X=P / P_{\mathrm{s}}$ and $P_{\mathrm{s}}$ is defined by Equation (8). We find that with a given intensity of an amplifier system $\left(11.4 \mathrm{~W} / \mathrm{cm}^{2}\right)$ and molecular cross sections taken from Table 1 at $780 \mathrm{~nm}$, saturation parameter is $X=4.5$, and the resulting $k_{1}=(2.9 \pm 0.9) \times 10^{-3} \mathrm{~s}^{-1}$, that is virtually the same as measured directly in [28]. This result demonstrates once again that the multiphoton bleaching rate does not depend on local environment of mCherry ( $E$. coli cell or buffer). It is also not sensitive to the laser repetition rate even at high laser intensities (i.e., in super-quadratic regime).

\subsection{Multiphoton Bleaching of mPlum in E. coli Colonies}

Figure 5 shows the dependence of photobleaching rate on power for mPlum at $790 \mathrm{~nm}$. Similar to mCherry, it shows a "bending" from the $\sim P^{2}$ to $\sim P^{4}$ law occurring at $P^{*}=45 \mathrm{~mW}$ $\left(I_{0}{ }^{*}=5.2 \times 10^{28}\right.$ photon $\left./ \mathrm{cm}^{2} / \mathrm{s}\right)$. It has been previously shown that mPlum, similarly to mCherry, bleaches at this wavelength and at $P=40-150 \mathrm{~mW}$ as a result of four-photon induced electron detachment [28]. The vertical photodetachment energy $(5.61 \mathrm{eV})$ requires photons with $\lambda<880 \mathrm{~nm}$ to initiate the process.

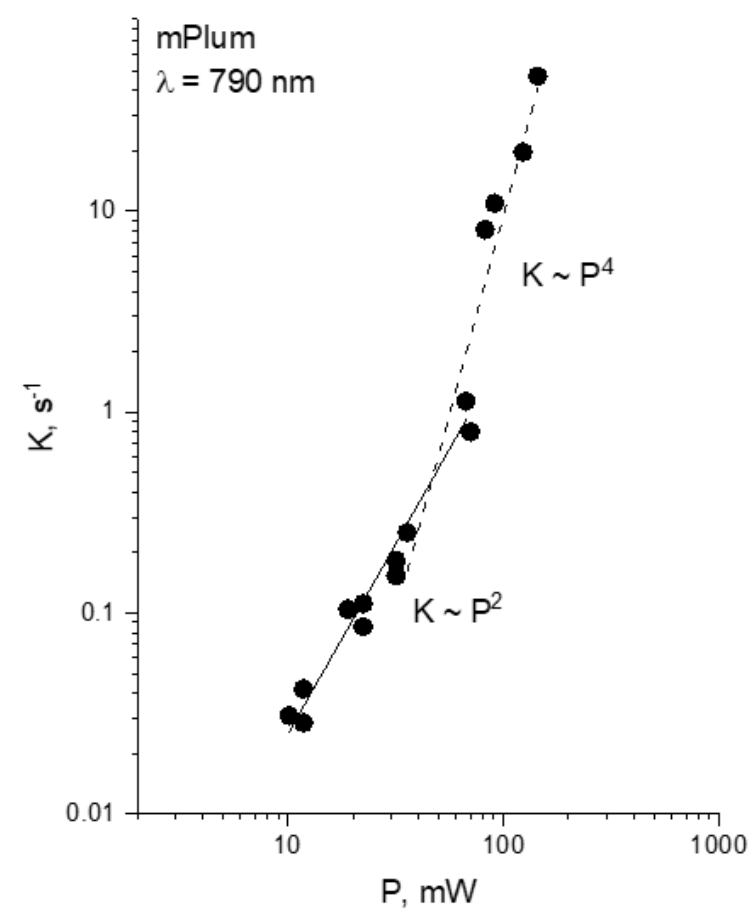

Figure 5. Power dependence of the initial bleaching rate of mPlum at $790 \mathrm{~nm}$, presented in double logarithmic scale.

However, the quadratic part has not been observed before [28] because of the used laser powers were above the threshold. We applied the same approach as above to obtain bleaching parameters for both the quadratic and fourth-power regimes (Supplementary Figures S11 and S12). The quantum yield of the two-photon induced reaction is $\varphi_{\mathrm{m}}=(1.3 \pm 0.2) \times 10^{-4}$ (i.e., of the same order as for mCherry in this spectral region). The product of the two consecutive processes cross sections is, $\sigma_{\mathrm{mn}} \sigma_{\mathrm{nc}}=(1.6 \pm 0.2) \times 10^{-35} \mathrm{~cm}^{4}$, that is also close to the value obtained for mCherry at $780 \mathrm{~nm}$. 


\subsection{Multiphoton Bleaching of jREX-GECO1 in E. coli Colonies}

JREX-GECO1 is a new red genetically encoded $\mathrm{Ca}^{2+}$ indicator, described in [35]. In a $\mathrm{Ca}^{2+}$-free state, its deprotonated chromophore is weakly fluorescent. Upon binding $\mathrm{Ca}^{2+}$, the chromophore becomes protonated and acquires strong fluorescence with a large Stokes shift. It was interesting to see in what state it is present in E. coli cells and characterize its multiphoton bleaching properties.

Figure 6 shows that the 2PE spectrum of jREX-GECO1 in E. coli cells (circles) closely follows the spectrum of the $\mathrm{Ca}^{2+}$-bound state in solution (purple line). This result suggests that in $E$. coli bacteria there is enough $\mathrm{Ca}^{2+}$ to saturate the sensor.

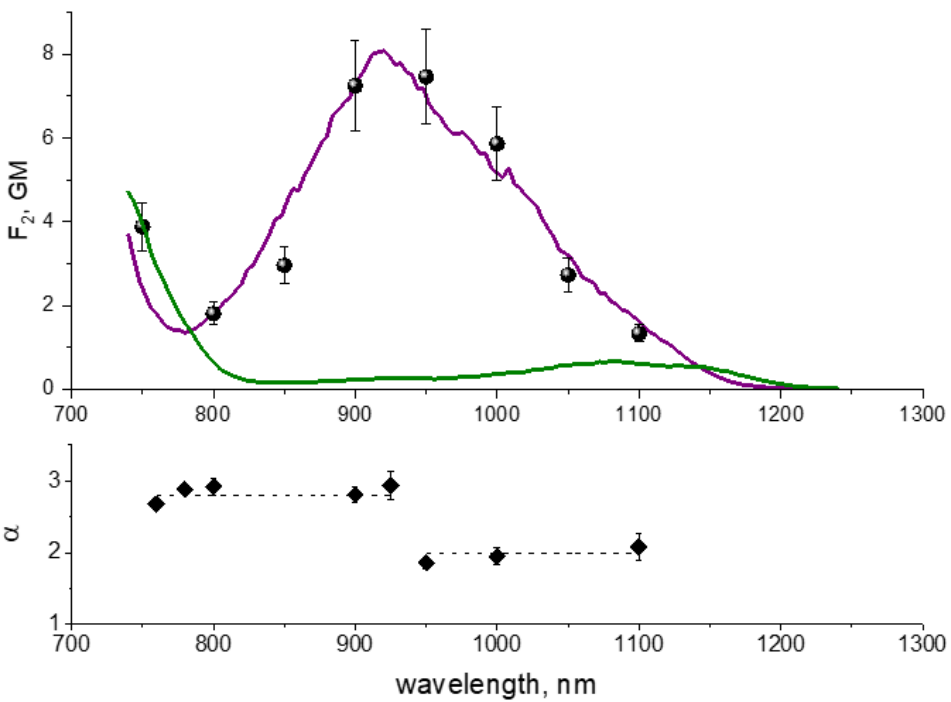

Figure 6. Top: Two photon action spectra of jREX-GECO1 measured in $\mathrm{Ca}^{2+}$-free (green line) and $\mathrm{Ca}^{2+}$-bound (purple line) states in buffer [35] and in E. coli cells (normalized to $\mathrm{Ca}^{2+}$-bound spectrum at $900 \mathrm{~nm}$ ). Bottom: Power exponent of the bleaching rate power dependence as a function of wavelength.

The nature of the broad 2PE band peaking at $~ 920 \mathrm{~nm}$ is not completely understood, but it looks similar to that observed in the one-photon absorption spectrum, peaking at $\sim 490 \mathrm{~nm}$ [35]. It is assumed that it mainly belongs to a neutral chromophore. However, unusually broad wing at 1000-1100 nm suggests that the whole band could contain two or more hidden electronic transitions [35].

The power dependences of photobleaching rates are very different as compared to mCherry and mPlum. For the wavelengths longer than $925 \mathrm{~nm}$, they are quadratic in the range of $\sim 10-70 \mathrm{~mW}$, see Figure $7 \mathrm{a}$. At $P>70 \mathrm{~mW}$, they start growing faster, but we were not able to determine a power exponent for this region because of insufficient range of available powers.

From the quadratic segments of power dependences, we obtained photochemical quantum yields $\varphi_{1}$ by using the same procedure as in Section 2.2 (see Supplementary Figures S13-S15 for the fits). The results show that the quantum yield at $1100 \mathrm{~nm}$ is higher than that at $950-1000 \mathrm{~nm}$ by a factor of $\sim 4$, Table 3 . This can be due to a presence of a small fraction of anionic chromophore dominating the absorption at $1100 \mathrm{~nm}$, and bleaching faster than the neutral form (that mainly absorbs at 950-1000 nm). 

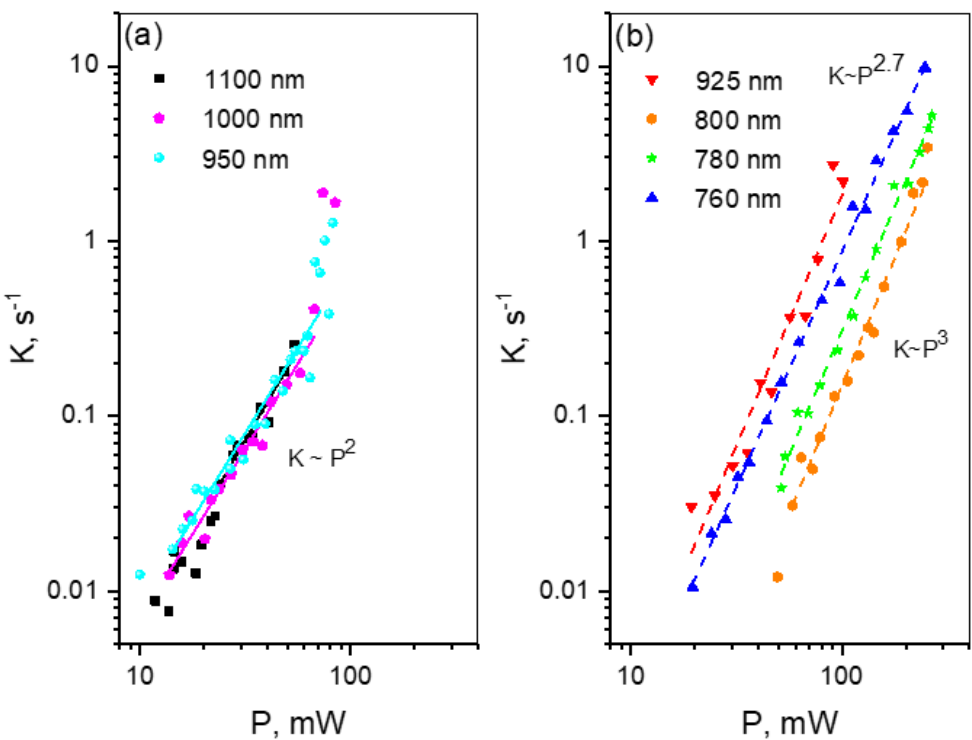

Figure 7. Power dependences of the bleaching rates of jREX-GECO1 at different wavelengths presented in double logarithmic scale. (a) Long wavelength range; (b) Short wavelength range.

Table 3. Molecular photobleaching parameters of jREX-GECO1 at different wavelengths.

\begin{tabular}{|c|c|c|c|c|c|c|}
\hline$\lambda, \mathrm{nm}$ & $n$ & $\sigma_{2}, \mathrm{GM}^{(\mathrm{a})}$ & $\varphi_{1}^{(b)}$ & $\sigma_{\mathrm{mnn}} \varphi_{\mathrm{n}}, \mathrm{cm}^{2}(\mathrm{c})$ & $\sigma_{\mathrm{mn}}, \mathrm{cm}^{2(\mathrm{~d})}$ & $\varphi_{\mathrm{n}}$ \\
\hline 1100 & 2 & 7.9 & $(2.1 \pm 0.3) \times 10^{-4}$ & - & - & - \\
\hline 1000 & 2 & 25 & $(5.7 \pm 0.9) \times 10^{-5}$ & - & - & - \\
\hline 950 & 2 & 34 & $(5.0 \pm 0.8) \times 10^{-5}$ & - & - & - \\
\hline 925 & 3 & 38 & - & $(1.2 \pm 0.2) \times 10^{-20}(\mathrm{~m}=1)$ & - & - \\
\hline 900 & 3 & 35 & & $(2.0 \pm 0.3) \times 10^{-20}(\mathrm{~m}=1)$ & - & - \\
\hline 800 & 3 & 8.8 & - & $(7.7 \pm 1.1) \times 10^{-21}(\mathrm{~m}=1)$ & - & - \\
\hline 780 & 3 & 6.5 & - & $(1.5 \pm 0.2) \times 10^{-20}(\mathrm{~m}=1)$ & - & - \\
\hline 760 & 3 & 8.7 & - & $(3.0 \pm 0.5) \times 10^{-20}(\mathrm{~m}>1)$ & $(1.4 \pm 0.4) \times 10^{-17}$ & $(2.2 \pm 0.7) \times 10^{-3}$ \\
\hline
\end{tabular}

Column 2 shows the number of photons $n$ involved in the process. (a) $2 \mathrm{PA}$ cross sections of $\mathrm{Ca}^{2+}$-saturated

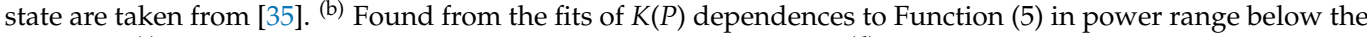
threshold. ${ }^{(c)}$ Found from the fits of $K(P)$ dependences to Function (6). ${ }^{(d)}$ Found from the fits of $K(P)$ dependences to Function (7).

Upon passing to a shorter wavelength range, a sharp threshold near $\lambda^{*} \approx 950 \mathrm{~nm}$ is observed where the quadratic dependence switches to super-quadratic, see Figure 6 , bottom, and Figure $7 \mathrm{~b}$. In contrast to mFruits (mCherry and mPlum) where the power exponent is larger than 3 in this spectral region, in jREX-GECO1 it is equal or less than 3. Therefore, we assume that the reaction requires only three photons, not four, and proceeds through a bound electronic state without reaching photodetachment continuum boundary. The wavelength threshold at $950 \mathrm{~nm}$ is probably related to a specific resonance where the photon energy becomes sufficient for excitation of this bound state via the $S_{1} \rightarrow S_{n}$ transition. We believe that the photobleaching mechanism in jREX-GECO1 is different from that in mFruits because the chromophore in the former is neutral (protonated) compared to anionic (deprotonated) one in mFruits. The protonation of chromophore in jREX-GECO1 is facilitated by the substitution of neutral amino acid residue at position 163 (Gln in mCherry or Met in mPlum) with negative Glu (see Supplementary Figure S37 and Table S2) that supposedly comes to a close contact with the chromophore phenolate oxygen in $\mathrm{Ca}^{2+}$-bound state. Since the electron detachment energy is much higher for the protonated chromophore than for the deprotonated one, because of strong electrostatic interaction between the 
electron and remaining molecular frame in the former, the four-photon detachment becomes forbidden there by energy conservation.

The power dependences at 780-925 nm, plotted as $K$ vs. $P^{3}$, were fitted to Equation (6) (Supplementary Figures S16-S18) to obtain the parameter $\sigma_{\operatorname{mn}} \varphi_{\mathrm{n}}$ from the slope:

$$
a_{3}=\frac{4}{5 \pi^{3}} \sigma_{2} \sigma_{\operatorname{mn}} \varphi_{\mathrm{n}} \frac{\Gamma_{4}(\eta)}{\Delta \tau f^{2}(h v)^{3} w_{0}^{6}}
$$

The values of $\sigma_{\mathrm{mn}} \varphi_{\mathrm{n}}$ do not change systematically with the wavelength and fall in the range $(0.8-2.0) \times 10^{-20} \mathrm{~cm}^{2}$. The power dependence at $760 \mathrm{~nm}$ is slightly saturated, $K \sim P^{2.7}$ (if the whole range is used for fitting). This makes it possible to obtain parameters $\sigma_{\mathrm{mn}}$ and $\varphi_{\mathrm{n}}$ separately, see Table 3, by using fitting Function (7), (Supplementary Figure S19). The $760 \mathrm{~nm}$ excitation corresponds to a different transition $\left(\mathrm{S}_{0} \rightarrow \mathrm{S}_{\mathrm{m}}\right)$, compared to the one at $780-925 \mathrm{~nm}\left(\mathrm{~S}_{0} \rightarrow \mathrm{S}_{1}\right)$, see Figure 6 , and that can probably explain why the value of $\sigma_{\mathrm{mn}} \varphi_{\mathrm{n}}$ is higher at $760 \mathrm{~nm}$ than the corresponding average value in the 780-925 region.

\subsection{Multiphoton Bleaching of tdTomato in E. coli Colonies}

The 2PE spectral shape of tdTomato in E. coli cells overlaps with that previously measured in purified solution at pH8 [11], Figure 8, top, suggesting the expression of this protein in the cells.

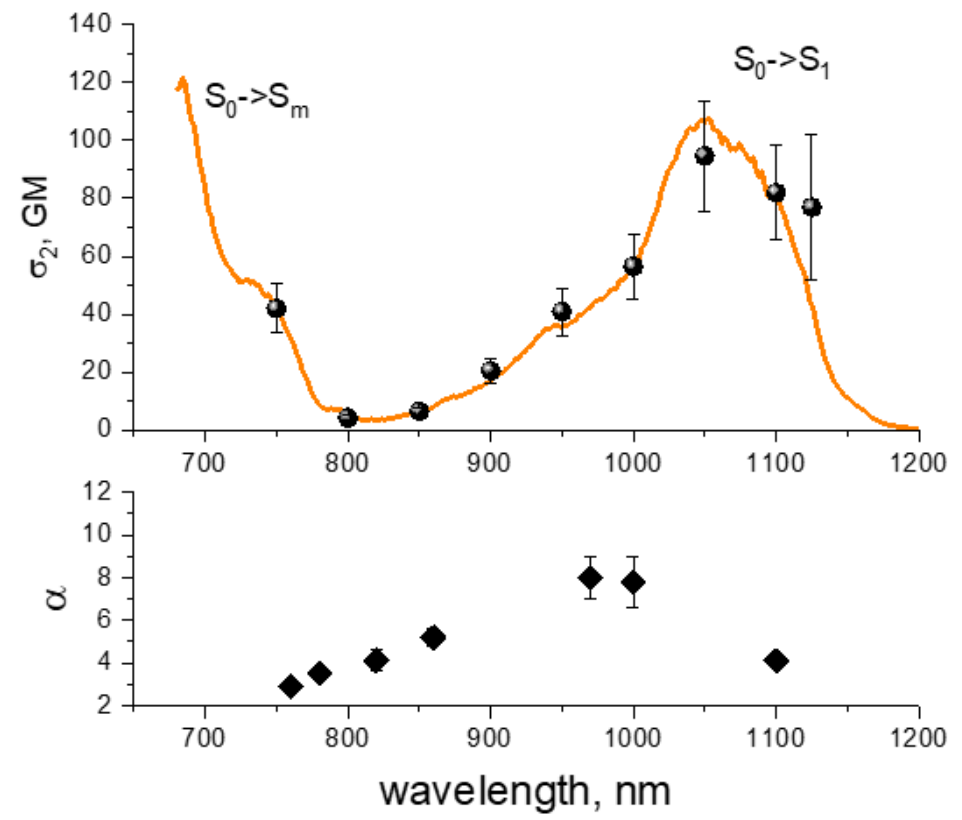

Figure 8. Top: Two-photon absorption spectrum of tdTomato in purified buffer solution at pH8, (orange line) [11] and two-photon excitation spectrum of tdTomato measured in E. coli cells (symbols) (normalized to the 2PA cross section of solution spectrum at $750 \mathrm{~nm}$ ). Bottom: Power exponent $\alpha$ of the dependence of photobleaching rate on laser power (see text for details).

Compared to other proteins, tdTomato shows the most diverse and unexpected multiphoton bleaching behavior. When excited at the long-wavelength side of the $S_{0} \rightarrow S_{1}$ transition at $1100 \mathrm{~nm}$, the power dependence follows a $\sim P^{4}$ law even at low power levels without any observable $P^{2} \rightarrow P^{4}$ transition, Figure 9a, black squares. This behavior is very different from mCherry, where the rate followed the quadratic law at $1100 \mathrm{~nm}$ in the same range of powers and the $P^{4}$ dependence emerged only for the wavelengths $\leq 800 \mathrm{~nm}$. This observation suggests that the four-photon electron detachment energy lies much lower in tdTomato than in mCherry. We estimate the VDE $<4 h v=4.5 \mathrm{eV}$ (for $h v=1.13 \mathrm{eV}$, corresponding to $1100 \mathrm{~nm}$ ) for tdTomato vs. VDE $=5.5 \mathrm{eV}$ in mCherry. Physically a decrease in VDE can mean that the electrostatic environment of the chromophore in tdTomato is less 
electro-positive than in mCherry. The main differences in electrostatic environment of the chromophore in RFPs are due to the charges of residues of amino acids 83, 163, and 215 and their distances from the chromophore [28] (Supplementary Figure S37 and Table S2). In mCherry, residues L83 and E215 are both neutral (E215 is protonated [38] at pH7), and in tdTomato K83 is positive and E215 is negative (deprotonated, according to our alkaline titration experiment, not shown). Although the total charge of the two residues is zero in both proteins, the negative charge of E215 in tdTomato is much closer to the chromophore than the positive charge of K83 (Table S2), which makes the environment of tdTomaato less positive than mCherry. Plotting of $K$ vs. $P^{4}$ (Supplementary Figure S20) makes it possible to find the value of the product $\sigma_{\mathrm{mn}} \sigma_{\mathrm{nc}}$ at this wavelength, see Table 4.
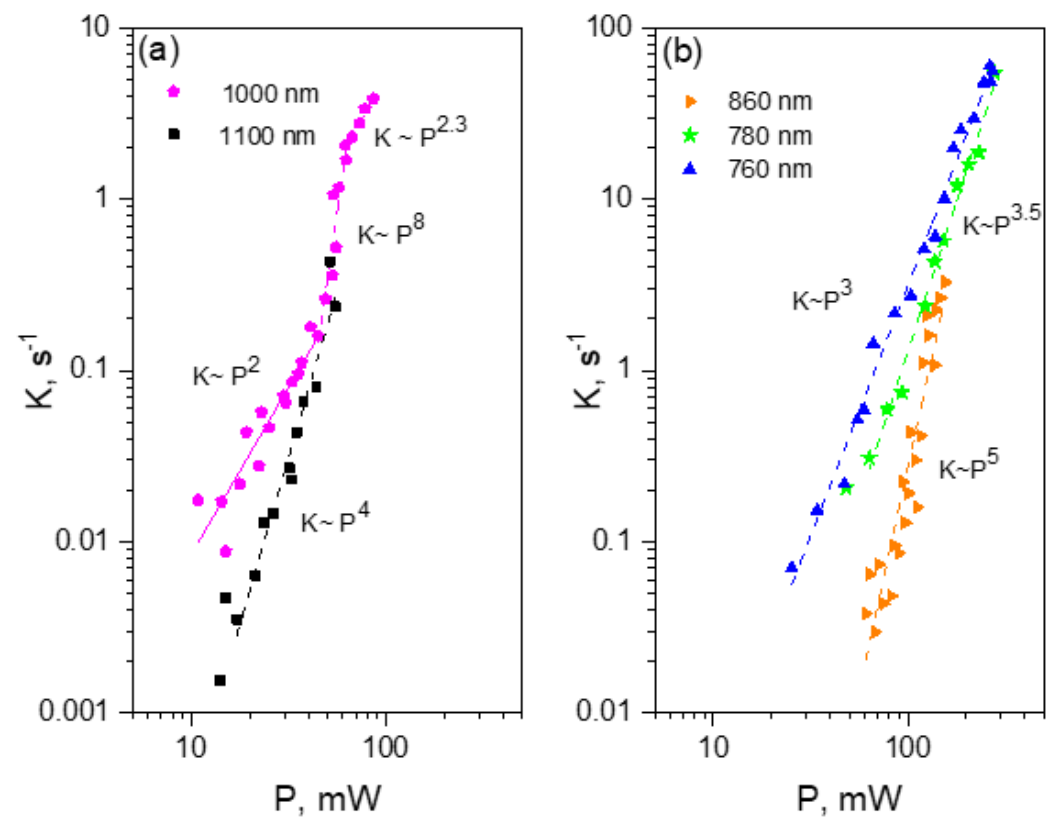

Figure 9. Power dependences of the bleaching rates of tdTomato at different wavelengths (see legends in the insets) presented in double logarithmic scale. (a) Long wavelength range; (b) Short wavelength range.

Table 4. Molecular photobleaching parameters of tdTomato at different wavelengths.

\begin{tabular}{|c|c|c|c|c|c|c|}
\hline$\lambda, \mathrm{nm}$ & $\mathbf{n}$ & $\sigma_{2}, \mathrm{GM}^{(\mathrm{a})}$ & $\varphi_{1, \mathrm{~m}}{ }^{(\mathbf{b})}$ & $\sigma_{\mathrm{mn}} \sigma_{\mathrm{nc}}, \mathrm{cm}^{4(\mathrm{c})}$ & $\sigma_{\mathrm{mn}}, \mathrm{cm}^{2(\mathrm{~d})}$ & $\sigma_{\mathrm{nc}}, \mathrm{cm}^{2}(\mathrm{~d})$ \\
\hline 1100 & 4 & 80 & - & $(2.3 \pm 0.4) \times 10^{-36}$ & - & - \\
\hline 1000 & 2 to 8 & 56 & $(3.7 \pm 0.6) \times 10^{-5}$ & - & - & - \\
\hline 970 & 2 to 10 & 42 & $(1.6 \pm 0.2) \times 10^{-5}$ & - & & \\
\hline 860 & 5 & 22 & - & - & - & - \\
\hline 820 & 4 & 3.2 & - & $(4.3 \pm 0.2) \times 10^{-36}$ & - & - \\
\hline 780 & 4 & 8.7 & - & $(1.6 \pm 0.8) \times 10^{-35}$ & $(4.3 \pm 1.5) \times 10^{-17}$ & $(3.8 \pm 1.3) \times 10^{-20}$ \\
\hline 760 & $4(\alpha=3)$ & 31 & - & - & - & $(4.8 \pm 0.7) \times 10^{-19}$ \\
\hline
\end{tabular}

Column 2 shows the number of photons $n$ involved in the process. (a) 2PA cross sections are taken from [11] (b) Found from the fits of $K(P)$ dependences to Function (5) in power range below the threshold. (c) Found from the fits of $K(P)$ dependences to Function (9). ${ }^{(d)}$ Found from the fits of $K(P)$ dependences to Function (10).

At shorter wavelengths, 970-1000 nm, the photobleaching rate changes very dramatically as a function of power, see Figure 9a for $1000 \mathrm{~nm}$ data (magenta pentagons) and Supplementary Figure S21 for $970 \mathrm{~nm}$ data. First, at $P=10-40 \mathrm{~mW}$ the dependence is quadratic (Supplementary Figure S22), with the quantum yields of the reaction starting from $S_{1}$ state similar to those of mCherry $\left(\varphi_{1} \sim 10^{-5}\right)$, see Table 4 .

As the laser power exceeds $P^{*}=45 \mathrm{~mW}\left(I_{0}^{*}=6.5 \times 10^{28}\right.$ photon $\left./ \mathrm{cm}^{2} / \mathrm{s}\right)$, the dependence suddenly becomes extremely sharp, following the power law with the exponent 
$\alpha=8 \pm 1$. To the best of our knowledge, the photobleaching with the involvement of such a high number of photons has not been observed for any organic molecule. Interestingly, the initial (unbleached) fluorescence signal follows a perfect quadratic dependence, Supplementary Figure S23. This suggests that other spurious processes, like harmonic or white continuum generation, can be excluded and most probably the observed kinetics are due to photochemical reaction. When the power reaches $\sim 60 \mathrm{~mW}$, the dependence quickly saturates and becomes either quadratic or slightly super-quadratic, $(\alpha \approx 2.3 \pm 0.3)$. This suggests that all the electronic transitions involved in the ladder-type excitation saturate, including the last one that leads to ionization.

An intriguing question of why the four-photon electron detachment observed at longer wavelengths $(1100 \mathrm{~nm})$ turns to a much higher-order process at shorter wavelengths remains open and deserves further investigation. We can only speculate that in tdTomato the chromophore can co-exist in the anionic and neutral forms. The anionic form has the 2PE maximum at $\sim 1050-1100 \mathrm{~nm}$ and bleaches according to a $K \sim P^{4}$ law. The neutral chromophore, with the 2PE at 900-1000 nm (corresponding to a shoulder at $950 \mathrm{~nm}$ in Figure 8, top), bleaches according to a $K \sim P^{8}$ law. The RFP chromophore in its protonated (neutral) form has much larger vacuum electron detachment energy compared to deprotonated (anionic) form, $\mathrm{VDE}=7.64 \mathrm{vs}$. $3.27 \mathrm{eV}$, respectively [39]. The first value translates into the number of photons $n=\mathrm{VDE} / h v=6.2$, with $h v=1.24 \mathrm{eV}$ for the $1000 \mathrm{~nm}$ photon. This suggests that at least seven photons are needed to ionize the RFP chromophore in vacuum, close to our observation. Slightly positive chromophore surrounding in tdTomato, that electrostatically interacts with the positive molecular frame left after ionization, can further increase the VDE, and consequently $n$. We estimate that an electrostatic energy of $\sim 1.2 \mathrm{eV}$ would be enough to make $n=8$.

At shorter wavelengths, $760-820 \mathrm{~nm}$, the power dependence reflects the $n=4$ process with moderate $(\alpha=3.5$ at $780 \mathrm{~nm})$ or strong $(\alpha=3.0$ at $760 \mathrm{~nm})$ saturation. At these wavelengths, the anionic specie probably dominates in the 2PA spectrum. The corresponding values for the $\sigma_{\mathrm{mn}}$ and $\sigma_{\mathrm{nc}}$ cross sections are obtained (Supplementary Figures S24-S26) and presented in Table 4. At an intermediate wavelength of $860 \mathrm{~nm}$, we observe the power exponent $\alpha \approx 5$, which possibly reflects a competition between the bleaching of anionic and neutral chromophore species. The dependence of $\alpha$ on wavelength, Figure 8 bottom, shows a resonance at $950-1000 \mathrm{~nm}$, probably depicting the position of neutral chromophore 2PA maximum. It is interesting that a recent paper [40] found that the neutral chromophore of tdTomato has a one-photon absorption peak in the same spectral region, at $\sim 480 \mathrm{~nm}$. However, it is not clear yet to what extent the neutral specie can contribute to the 2PE spectrum.

\section{Discussion}

Our results show that even within a set of red FPs with the same chemical structure of the chromophore, the mechanisms of multiphoton bleaching are quite different. This includes different numbers of photons involved in the process and different power dependences observed at various excitation wavelengths even for a particular protein. Nevertheless, we try to compare here the performance of one protein at different wavelengths or different proteins at similar wavelength using a specific "figure of merit" (FOM) that reflects the maximum possible number of fluorescence photons collected from an FP during a given pixel dwell time in a TPLM experiment. The FOM parameter involves the molecular fluorescence brightness and photobleaching parameters (see Supplementary Information for derivation of equations). In the case of quadratic power dependence of photobleaching rate, the FOM is equal to the ratio of fluorescence quantum yield and photochemical reaction quantum yield starting either from state 1 or $\mathrm{m}$ (see Figure $1 \mathrm{a}, \mathrm{b}$ ):

$$
\operatorname{FOM}^{(2)}=\frac{\varphi_{\mathrm{F}}}{\varphi_{1, \mathrm{~m}}}
$$


For the third-order process without saturation, Figure 1c, the FOM reads

$$
\mathrm{FOM}^{(3)}=\frac{\varphi_{\mathrm{F}} \sigma_{2}^{1 / 3}}{\left(\sigma_{\mathrm{mn}} \varphi_{\mathrm{n}}\right)^{2 / 3}}
$$

In the fourth-order process without saturation, we have

$$
\mathrm{FOM}^{(4)}=\frac{\varphi_{\mathrm{F}} \sigma_{2}^{1 / 2}}{\left(\sigma_{\mathrm{mn}} \sigma_{\mathrm{nc}}\right)^{1 / 2}} .
$$

Using these equations, we estimated the FOM values of the fourth-order process observed experimentally for mCherry, mPlum, and tdTomato at several wavelengths, see Table 5. It should be kept in mind that the comparison between different wavelengths and different proteins using these numbers is possible only for the same laser peak photon flux and the same mechanism of the process.

Table 5. Figure of Merit for TPLM applications of FPs showing fourth-order power dependence of

\begin{tabular}{|c|c|c|c|c|c|}
\hline Protein & $\lambda, \mathrm{nm}$ & $\sigma_{2}, \mathrm{GM}^{(\mathrm{a})}$ & $\sigma_{\mathrm{mn}} \sigma_{\mathrm{nc}}, \mathrm{cm}^{4}$ & $\varphi_{\mathrm{F}}{ }^{(b)}$ & $\operatorname{FOM}^{(4)}\left[\times 10^{-8}\right]$ \\
\hline \multirow[t]{3}{*}{ mCherry } & 800 & 22 & $5.0 \times 10^{-36}$ & 0.24 & 5 \\
\hline & 780 & 42 & $1.7 \times 10^{-35}$ & 0.24 & 3.8 \\
\hline & 760 & 71 & $3.3 \times 10^{-35}$ & 0.24 & 3.5 \\
\hline mPlum & 790 & 43 & $1.6 \times 10^{-35}$ & 0.13 & 2.1 \\
\hline \multirow[t]{3}{*}{ tdTomato } & 1100 & 80 & $2.3 \times 10^{-36}$ & 0.72 & 43 \\
\hline & 820 & 3.2 & $4.3 \times 10^{-36}$ & 0.72 & 6.2 \\
\hline & 780 & 8.7 & $1.6 \times 10^{-35}$ & 0.72 & 5.3 \\
\hline
\end{tabular}
multiphoton bleaching rate.

(a) Taken from [11]. ${ }^{(b)}$ Taken from [10].

For the $\mathrm{S}_{0} \rightarrow \mathrm{S}_{\mathrm{m}}$ transition, excitation of mCherry at $800 \mathrm{~nm}$ is slightly beneficial, compared to $780 \mathrm{~nm}$, because of smaller $\sigma_{\mathrm{mn}} \sigma_{\mathrm{nc}}$ parameter for the former. At $790 \mathrm{~nm}$, mPlum shows about two-times smaller FOM ${ }^{(4)}$ compared to mCherry because of proportionally smaller fluorescence quantum yield. tdTomato has the FOM ${ }^{(4)}$ similar to mCherry at $780-820 \mathrm{~nm}$, but much larger at $1100 \mathrm{~nm}$. This strong enhancement is due to much higher 2PA cross section of tdTomato compared to mCherry at this wavelength.

Table 6 presents the FOM ${ }^{(2)}$ values obtained for the RFPs under study in the power ranges where the bleaching rate follows quadratic power dependence.

Table 6. Figure of Merit for TPLM applications of FPs showing second-order power dependence of multiphoton bleaching rate.

\begin{tabular}{ccccc}
\hline Protein & $\lambda, \mathbf{n m}$ & $\boldsymbol{\varphi}_{\mathbf{1 , \mathbf { m }}}{ }^{(\mathbf{a})}$ & $\boldsymbol{\varphi}_{\mathbf{F}}{ }^{(\mathbf{b})}$ & $\mathbf{F O M}^{(\mathbf{2})}\left[\times \mathbf{1 0}^{\mathbf{3}}\right]$ \\
\hline mCherry & 1100 & $1.6 \times 10^{-5}$ & 0.24 & 15 \\
& 1000 & $1.0 \times 10^{-4}$ & 0.24 & 2.4 \\
& 800 & $1.0 \times 10^{-4}$ & 0.24 & 2.4 \\
& 780 & $8.5 \times 10^{-5}$ & 0.24 & 2.8 \\
mPlum & 760 & $1.0 \times 10^{-4}$ & 0.24 & 2.4 \\
jREX-GECO1 & 790 & $1.3 \times 10^{-4}$ & 0.13 & 1 \\
tdTomato & 1100 & $2.1 \times 10^{-4}$ & 0.21 & 3.7 \\
& 1000 & $5.7 \times 10^{-5}$ & 0.21 & 4.2 \\
& 950 & $5.0 \times 10^{-5}$ & 0.21 & 20 \\
& 1000 & $3.7 \times 10^{-5}$ & 0.72 & 45 \\
\hline
\end{tabular}

(a) Taken from [11]. ${ }^{(b)}$ For mFruits and tdTomato taken from [10] and for jREX-GECO1—from [35]. 
In this regime, mCherry has much higher $\mathrm{FOM}^{(2)}$ at $1100 \mathrm{~nm}$ than at $760-1000 \mathrm{~nm}$. This is explained by much lower $\varphi_{1}$ for the reaction starting from the $S_{1}$ level relatively to $S_{m}$ level. Compared to mCherry, mPlum shows lower FOM ${ }^{(2)}$ at $790 \mathrm{~nm}$, and jREXGECO1-higher at 950-1000 nm. Excitation of tdTomato with low powers at 970-1000 nm (Figure 9a), provides extremely large relative $\mathrm{FOM}^{(2)}$ values thanks to high fluorescence quantum yield and relatively low photobleaching yield.

In summary, we developed a method of quantitative characterization of multiphoton bleaching of FPs expressed in E. coli colonies that one can use to compare bleaching rates of different mutants in standardized experimental conditions. Our physical model proposed in [28] and further developed here, makes it possible to obtain key molecular parameters involved in photobleaching process. Knowing laser parameters, such as wavelength, pulse duration and peak photon flux in focal plane in addition to molecular bleaching parameters, makes it possible to predict the bleaching rate in a specific experiment. In addition to quantitative estimations of FOM provided above, we can further suggest some general, qualitative, approaches to reduce the bleaching rates. We have shown here, for a first time to the best of our knowledge, that there is often a range of laser photon fluxes where the power dependence of bleaching follows quadratic law. After a critical threshold, $P=P^{*}$, is passed, the dependence turns to a much faster, super-quadratic one that was observed in several previous studies. We suggest that in the TPLM experiments the working laser intensity should be selected close to this threshold to provide the best SBR. We also found that, in agreement with previous observations [20-22], it is more advantageous (in terms of brightness / photobleaching ratio) to excite RFPs at their longer wavelength transition, $\mathrm{S}_{0} \rightarrow \mathrm{S}_{1}$ (near 1050-1100 nm). In particular, in the case of mCherry, excitation at $1100 \mathrm{~nm}$ does not activate a detrimental four-photon electron photodetachment process, and the power dependence follows a quadratic law where any increase in laser intensity provides better SBR ratio, but does not change the brightness/photobleaching rate ratio. At shorter wavelengths, corresponding to a higher, $\mathrm{S}_{0} \rightarrow \mathrm{S}_{\mathrm{m}}$ transition $(760-800 \mathrm{~nm})$, there exists a laser intensity threshold, specific for each wavelength, where the power dependence switches from quadratic to much faster law, corresponding to four-photon process. Such switching was also observed for mPlum.

For the new, red genetically-encoded $\mathrm{Ca}^{2+}$-sensor, jREX-GECO1, with a long Stokes shift in the $\mathrm{Ca}^{2+}$-bound state, we observe slow photobleaching, following quadratic power law in a broad spectral region from $950-1100 \mathrm{~nm}$. At shorter wavelengths, $\lambda<950 \mathrm{~nm}$, the bleaching rate starts to grow faster, i.e., according to a third power law. Therefore, excitation at these wavelengths should be avoided.

One of the brightest under two-photon excitation red FP, tdTomato, shows unexpected power dependences of the bleaching rate at some particular wavelengths. At the longest wavelength studied, $\lambda=1100 \mathrm{~nm}$, the bleaching follows a $K \sim P^{4}$ dependence, reflecting four-photon process. At $970-1000 \mathrm{~nm}$, the dependence starts as quadratic, and then after passing some critical power, becomes very sharp, following the $K \sim P^{8}$ law. At even higher powers, it quickly saturates to become close to quadratic again. At shorter wavelength region, $760-820 \mathrm{~nm}$, the power dependence follows the $K \sim P^{4}$ law with some signs of saturation, typical for mCherry and mPlum. We tentatively explain these observations by a presence of two forms of the chromophore in tdTomato, with the neutral form dominating in the region of $950-1000 \mathrm{~nm}$.

\section{Materials and Methods}

In this work, we studied multiphoton bleaching of RFPs expressed in E. coli cells. The cells make colonies on the surface of agar in a square Petri dish. Typically, a colony measures $\sim 1 \mathrm{~mm}$ in diameter and $\sim 0.2 \mathrm{~mm}$ in thickness. The rod-shaped E. coli cell is $\sim 2 \mu \mathrm{m}$ long with a diameter of $<1 \mu \mathrm{m}$. 


\subsection{Expressing RFPs in E. coli Colonies}

His-tagged RFPs were expressed in DH10B E. coli cells with bacterial expression plasmids encoding each protein. mCherry was encoded on the $\mathrm{pNCS}$ vector, which contains a promoter for constitutive expression. tdTomato, mPlum, and jREX-GECO1 were encoded on pBAD. tdTomato-pBAD was a gift from Michael Davidson and Nathan Shaner and Roger Tsien (Addgene plasmid \# 54856; http:/ / n2t.net/addgene:54856 (accessed on 23 December 2021); RRID:Addgene_54856), mPlum-pBAD was a gift from Michael Davidson and Roger Tsien (Addgene plasmid \# 54564; http:/ / n2t.net/addgene:54564 (accessed on 23 December 2021); RRID:Addgene_54564). jREX-GECO1 was grown into a modified pBAD backbone pTorPE with restriction cloning. jpTorPE-jREX-GECO1 was a gift from Robert Campbell and Thomas Hughes (Addgene plasmid \# 113941; http:/ / n2t.net/addgene:113941 (accessed on 23 December 2021); RRID:Addgene_113941). E. coli expressing an RFP were cultured in Terrific Broth (BD, Sparks, MD, USA) for $18 \mathrm{~h}$ at $37^{\circ}$. To induce expression of the $\mathrm{pBAD}$ plasmids, $0.1 \% w / v$ of L-arabinose was added to the media before inoculating.

\subsection{Preparing Plates with E. coli Colonies}

Square $10 \times 10 \mathrm{~cm}$ Petri dishes (Simport, Beloeil, QC, Canada) were filled with $80 \mathrm{~mL}$ of $\mathrm{LB}$ agar at $50^{\circ} \mathrm{C}$ with the appropriate antibiotic and, in the case of $\mathrm{pBAD}$ vector expression, $0.02 \% w / v$ of L-arabinose added. The plates were set on a horizontal surface for $20 \mathrm{~min}$. Transformed E. coli suspension was diluted 1:10, 1:100, and 1:1000 times with Terrific Broth and were spread onto the plate using $3 \mathrm{~mm}$ glass beads (Fisher Scientific, Walthman, MA, USA), and then the plates were incubated for $20 \mathrm{~h}$ at $37^{\circ} \mathrm{C}$. After incubation, the plates with colonies were put in refrigerator at $4{ }^{\circ} \mathrm{C}$ for 2 days. After 2 days, the colonies acquired a color and fluorescence corresponding to a mature RFP chromophore and were ready for optical experiments. The plates with E. coli colonies could be stored at $4{ }^{\circ} \mathrm{C}$ for a few months without losing the ability to fluoresce.

\subsection{Optical Instrument}

The customized optical instrument, called the GIZMO, was previously designed and implemented for screening of large libraries of FP mutants for their two-photon brightness. It was described in detail in [41]. Its complete CAD model as well as software and analysis programs can be found at github.com/rosanamolina/gizmo-paper (accessed on 23 December 2021). Here we used this instrument to characterize multiphoton bleaching parameters of a particular FP expressed in several colonies on the same plate. Briefly, the GIZMO consists of two optical arms used for one- and two-photon excitation, respectively. The micrometer stage can move the sample (a Petri dish) between the two arms. While in the one-photon arm, a brief illumination of the dish with an LED array provides fluorescence of individual colonies, captured with a CCD camera. The $x$ and $y$ coordinates of each colony are then accurately represented in the image. The two-photon arm is a variant of two-photon laser scanning microscope. A femtosecond laser (Insight DeepSee, SpectraPhysics, Milpitas, CA, USA) beam first gets attenuated with a computer-controlled Pockels cell, then passing through two galvo mirrors, a scan lens, and a tube lens, hits the objective lens mounted on a piezo stage allowing to scan the focal spot of a laser in $z$-direction with a range of $400 \mu \mathrm{m}$. The image obtained at the one-photon arm is used to bring a particular colony into the laser focus, using the $x-y$ movement of the stage. The fluorescence is collected by the same objective and directed through a reflecting dichroic mirror and a combination of a $570-680 \mathrm{~nm}$ band pass and a $745 \mathrm{~nm}$ short pass filters to PMT.

\subsection{Photobleaching Experiments}

The customized photobleaching program (written in MATLAB) makes it possible to run photobleaching kinetics consecutively at six different spots $(x, y, z)$ inside one colony. We first selected a bright colony on the plate by using an image acquired in the one-photon arm, then in the two-photon arm moved the stage in the $x$ and $y$ directions, such that the center of this colony came to the laser beam axis. Next, we adjusted the $z$ position of the 
stage to get the strongest fluorescence signal. The photobleaching program calculated the coordinates $(x, y)$ of six points arranged in the apices of a hexagon with the diameter of $80 \mu \mathrm{m}$ around the initial point. At each point, the program found an optimum $z$ position (with maximum fluorescence signal) and then at this position recorded the fluorescence decay curve with a particular, program-controlled (with Pockels cell) laser power, and then moved sequentially to the next spots where it recorded the decay at different power levels. In most of the cases we used $10 \mathrm{~s}$ for a dwell time per point, but for some slow kinetics, $30 \mathrm{~s}$. Typically, the binning of 100 data points (with averaging) was used to get totally 25,000 points per $10 \mathrm{~s}$ of scan. The program runs on top of a legacy version of ScanImage (Vidrio Technologies, Ashburn, VA, USA). In these bleaching experiments we used a low numerical aperture objective lens, Plan-Neofluar $2.5 \times / 0.075$ (Carl Zeiss, White Plains, NY, USA). A low NA value helps to keep the laser waist diameter in focus (7-11 $\mu \mathrm{m})$, much larger than the size of an E. coli cell $(0.5-2 \mu \mathrm{m})$. Homogeneous irradiation of a cell allows disregarding of diffusion artifacts in quantitative description of bleaching kinetics. This particular lens has a good transmittance in NIR and its low NA causes less group velocity dispersion (GVD) [42].

\subsection{Characterizing Laser Beam Parameters in Focal Area}

The laser waist $w$ in focal area was measured with the razor blade method. The razor was fixed onto a GIZMO micron stage and the power meter was mounted underneath. The stage was moved in $x$ direction at a fixed $z$ position. The resulting dependence of power on $x$ was fit to a function

$$
P(x)=A \int_{-\infty}^{x} \exp \left(-\frac{2\left(t-x_{c}\right)^{2}}{w^{2}}\right) d t .
$$

This scan was performed at several positions of $z$, and the resulting dependence of $w$ on $z$ was plotted and fit to a function

$$
w(z)=w_{0} \sqrt{1+\left(\frac{\lambda\left(z-z_{0}\right)}{\pi w_{0}^{2}}\right)^{2}}
$$

The best-fit parameters $z_{0}$ and $w_{0}$ provide the focal plane position and the beam waist radius at focal plane, respectively. This procedure was repeated for several laser wavelengths, and the dependence of $w_{0}$ on wavelength is shown in Supplementary Figure S27. The resulting waist parameter $w_{0}$ varies between 3.5 and $5.5 \mu \mathrm{m}$. The Rayleigh length, $z_{R}=\pi w_{0}^{2} / \lambda$, was calculated and plotted as a function of $\lambda$, see Supplementary Figure S28. The focal spot size $\left(w_{0}\right)$ is a critical parameter in calculating multiphoton bleaching cross sections and quantum yields. The razor blade method used here could give a distorted $w_{0}$ value because of diffraction effects if the beam size on the back aperture of objective lens is not very small [43]. Therefore, we developed and employed an independent method of direct evaluation of $z_{\mathrm{R}}$ and $w_{0}$ in E. coli colonies, Section 4.6.

The pulse duration at $1100 \mathrm{~nm}$ was estimated as the transform-limited value for the Gaussian pulse shape. Using spectral widths measurements (Ocean Optics spectrometer, Ocean Insight, Rochester, NY, USA) of the laser output from 850 to $1000 \mathrm{~nm}$, we obtained an almost constant value of pulse duration, $\Delta \tau=(109 \pm 1) \mathrm{fs}$. We assume that the pulse duration after the beam expander and objective lens in our optical system does not acquire any GVD broadening at $1100 \mathrm{~nm}$ [42] and, therefore, is equal to $109 \mathrm{fs}$. To evaluate the relative pulse duration $\Delta \tau$ as a function of wavelength at the sample positions, we measured the two-photon excited fluorescence signal from a solution of Rhodamine $6 \mathrm{G}$ in methanol, cf. [23]. The solution, contained in a $1 \mathrm{~mm}$ thick optical cuvette, was placed in the focus of the objective lens and the power $P$ was kept constant when going from one wavelength to another. In this situation, the thickness of layer $l$ was much larger than the Rayleigh length, 
$l>>z_{R}$, and integrated fluorescence signal over time and space (taking into account the Gaussian-Lorentzian intensity distribution (2)), reads

$$
F_{2}=C \sigma_{2}(\lambda) \frac{\lambda}{\Delta \tau(\lambda)} P^{2},
$$

where $C$ is a constant. To find the pulse duration as a function of wavelength, we regroup the previous equation to obtain:

$$
\Delta \tau(\lambda)=C P^{2} \lambda \frac{\sigma_{2}(\lambda)}{F_{2}(\lambda)} .
$$

Figure S29 shows the $\Delta \tau$ values calculated according to this equation (with the $\sigma_{2}(\lambda)$ values taken from [44]), and normalized to the value of $109 \mathrm{fs}$ at $1100 \mathrm{~nm}$.

\subsection{Colony Thickness, Rayleigh Length, and Focal Spot Size inside a Colony}

To measure colony thickness and independently estimate the Rayleigh length, we performed a $z$-scan of fluorescence signal across several colonies expressing different FPs. This was done by changing the $z$ position with a step of 200 or $250 \mu \mathrm{m}$ (using the stage movement) and scanning a $400 \mu \mathrm{m}$ region with the piezo scan of the lens. Those $400 \mu \mathrm{m}$ segments were then "glued" together to obtain the $z$-profile of fluorescence intensity, Supplementary Figures S30-S35. These profiles were normalized to 1 and fitted to a model function that is obtained as a convolution of the Gaussian-Lorentzian distribution squared and a rectangular function of width $l$, describing the distribution of fluorophores inside a colony, see Supplementary Data. Colony thicknesses vary between 150 and $240 \mu \mathrm{m}$. The Rayleigh range obtained inside the colonies is $~ 30 \%$ larger than that in air, found in Section 5. We explain this effect by the dependence of wavelength of light on refractive index of medium. In fact, the general expression for $z_{R}$ in a medium reads [45]:

$$
z_{R}=\frac{n_{0} \pi w_{0}^{2}}{\lambda}
$$

where $n_{0}$ is the refractive index of medium and $\lambda$ is the wavelength of light in vacuum. Since the refractive index of water is 1.33 , the $z_{\mathrm{R}}$ measured in a colony should be 1.33 times larger than in air, as observed (Supplementary Figure S28). Finally, we calculate the $w_{0}$ values from the $z_{\mathrm{R}}$ measured in colonies, as

$$
w_{0}=\sqrt{\lambda z_{R} / n_{0} \pi} .
$$

The results match quite well those obtained with the razor blade method, see Figure S27.

\subsection{Two-Photon Excitation Spectra of RFPs inside E. coli Colonies}

To obtain relative shape of the 2PE spectrum of an RFPP inside a colony, we first measured its two-photon excited fluorescence signal $F_{2, \mathrm{~S}}(\lambda)$ (100 ms acquisition time, no bleaching) at several excitation wavelengths. In this measurement, position $z$ was adjusted to give the maximum signal (different at different $\lambda$ ). Then, exactly in the same excitation conditions (laser power) we measured the two-photon excited signal from the solution of Rhodamine 6G in methanol in $1 \mathrm{~mm}$ cuvette, $F_{2, \mathrm{R}}(\lambda)$. The cuvette was mounted inside an empty Petri dish and placed in focus of the laser instead of the plate with colonies. The $z$ position was again adjusted to the maximum signal. This sample was used as a reference to calibrate variations of the laser power and beam spatial and temporal profile as a function of wavelength. The corrected 2PE spectrum of an RFP sample in cells was then calculated according to

$$
f_{2 P E, S}(\lambda)=\frac{F_{2, S}(\lambda)}{F_{2, R}(\lambda)} f_{2 P E, R}(\lambda),
$$


where $f_{2 P E, R}(\lambda)$ is the corrected 2PE spectrum of the reference solution, presented in [44].

Supplementary Materials: The following supporting information can be downloaded at: https: / / www.mdpi.com/article/10.3390/ijms23020770/s1.

Author Contributions: Conceptualization, M.D.; Methodology, M.D. and R.S.M.; Software, J.F. and R.S.M.; Validation, M.D., R.S.M. and J.F.; Formal Analysis, M.D.; Investigation, M.D. and R.S.M.; Resources, M.D., R.S.M., and J.F.; Data Curation, M.D., R.S.M. and J.F.; Writing-Original Draft Preparation, M.D.; Writing-Review and Editing, M.D.; Visualization, M.D.; Supervision, M.D.; Project Administration, M.D.; Funding Acquisition, M.D. All authors have read and agreed to the published version of the manuscript.

Funding: This work was supported by the NINDS grants U01 NS094246 for MD, RSM, and JF and U24 NS109107 for MD and RSM.

Institutional Review Board Statement: Not applicable.

Informed Consent Statement: Not applicable.

Conflicts of Interest: The authors declare no conflict of interest.

\section{References}

1. Shaner, N.C.; Campbell, R.E.; Steinbach, P.A.; Giepmans, B.N.G.; Palmer, A.E.; Tsien, R.Y. Improved monomeric red, orange and yellow fluorescent proteins derived from Discosoma sp. red fluorescent protein. Nat. Biotechnol. 2004, 22, 1567-1572. [CrossRef]

2. $\quad$ Lin, M.Z.; McKeown, M.R.; Ng, H.-L.; Aguilera, T.A.; Shaner, N.C.; Campbell, R.E.; Adams, S.R.; Gross, L.A.; Ma, W.; Alber, T.; et al. Autofluorescent proteins with excitation in the optical window for intravital imaging in mammals. Chem. Biol. 2009, 16, 1169-1179. [CrossRef]

3. Chudakov, D.M.; Matz, M.V.; Lukyanov, S.; Lukyanov, K.A. Fluorescent proteins and their applications in imaging living cells and tissues. Physiol. Rev. 2010, 90, 1103-1163. [CrossRef]

4. Dana, H.; Mohar, B.; Sun, Y.; Narayan, S.; Gordus, A.; Hasseman, J.P.; Tsegaye, G.; Holt, G.T.; Hu, A.; Walpita, D.; et al. Sensitive red protein calcium indicators for imaging neural activity. eLife 2016, 5, e12727. [CrossRef] [PubMed]

5. Shen, Y.; Nasu, Y.; Shkolnikov, I.; Kim, A.; Campbell, R.E. Engineering genetically encoded fluorescent indicators for imaging of neuronal activity: Progress and prospects. Neurosci. Res. 2020, 152, 3-14. [CrossRef] [PubMed]

6. Denk, W.; Strickler, J.H.; Webb, W.W. Two-photon laser scanning fluorescence microscopy. Science 1990, 248, 73-76. [CrossRef]

7. Xu, C.; Zipfel, W.; Shear, J.B.; Williams, R.M.; Webb, W.W. Multiphoton fluorescence excitation: New spectral windows for biological nonlinear microscopy. Proc. Natl. Acad. Sci. USA 1996, 93, 10763-10768. [CrossRef]

8. Kazemipour, A.; Novak, O.; Flickinger, D.; Marvin, J.S.; Abdelfattah, A.S.; King, J.; Borden, P.M.; Kim, J.J.; Al-Abdullatif, S.H.; Deal, P.E.; et al. Kilohertz frame-rate two-photon tomography. Nat. Methods 2019, 16, 778-786. [CrossRef]

9. Lu, R.; Liang, Y.; Meng, G.; Zhou, P.; Svoboda, K.; Paninski, L.; Ji, N. Rapid mesoscale volumetric imaging of neural activity with synaptic resolution. Nat. Methods 2020, 17, 291-294. [CrossRef]

10. Drobizhev, M.; Makarov, N.S.; Tillo, S.E.; Hughes, T.E.; Rebane, A. Two-photon absorption properties of fluorescent proteins. Nat Methods 2011, 8, 393-399. [CrossRef] [PubMed]

11. Drobizhev, M.; Tillo, S.; Makarov, N.S.; Hughes, T.E.; Rebane, A. Absolute two-photon absorption spectra and two-photon brightness of orange and red fluorescent proteins. J. Phys. Chem. B 2009, 113, 855-859. [CrossRef] [PubMed]

12. Smith, A.M.; Mancini, M.C.; Nie, S. Bioimaging: Second window for in vivo imaging. Nat. Nanotechnol. $2009,4,710-711$. [CrossRef] [PubMed]

13. Nifosì, R.; Luo, Y. Predictions of novel two-photon absorption bands in fluorescent proteins. J. Phys. Chem. B 2007, 111, 14043-14050. [CrossRef] [PubMed]

14. Drobizhev, M.; Makarov, N.S.; Hughes, T.; Rebane, A. Resonance enhancement of two-photon absorption in fluorescent proteins. J. Phys. Chem. B 2007, 111, 14051-14054. [CrossRef]

15. Tillo, S.E.; Hughes, T.E.; Makarov, N.S.; Rebane, A.; Drobizhev, M. A new approach to dual-color two-photon microscopy with fluorescent proteins. BMC Biotechnol. 2010, 10, 6. [CrossRef]

16. Patterson, G.H.; Piston, D.W. Photobleaching in two-photon excitation microscopy. Biophys. J. 2000, 78, 2159-2162. [CrossRef]

17. Goodman, L.; Philis, J. Multiphoton Absorption Spectroscopy: Techniques, Instrumentation, and Applications. In Applied Laser Spectroscopy; Andrews, D.L., Ed.; VCH Publishers: New York, NY, USA, 1992; Chapter 8; pp. 319-364.

18. Lougnot, D.-J.; Goldschmidt, C.R. Photoionization of fluorescein via excited triplet and singlet states. J. Photochem. 1980, 12, 215-224. [CrossRef]

19. Redchenko, V.V.; Safronov, A.I.; Kirpichenok, M.A.; Grandberg, I.I.; Traven, V. Electronic Structure of $\pi$-Systems. XV. Photoelectron spectra of 7-aminocoumarin derivatives. J. Gen. Chem. USSR 1992, 62, 2313-2318.

20. Marchant, J.S.; Stutzmann, G.E.; Leissring, M.A.; LaFerla, F.M.; Parker, I. Multiphoton-evoked color change of DsRed as an optical highlighter for cellular and subcellular labeling. Nat. Biotechnol. 2001, 19, 645-649. [CrossRef] 
21. Robinson, L.C.; Marchant, J.S. Improved "optical highlighter" probes derived from discosoma red fluorescent protein. Biophys. J. 2005, 88, 1444-1457. [CrossRef]

22. Andresen, V.; Alexander, S.; Heupel, W.-M.; Hirschberg, M.; Hoffman, R.M.; Friedl, P. Infrared multiphoton microscopy: Subcellular-resolved deep tissue imaging. Curr. Opin. Biotechnol. 2009, 20, 54-62. [CrossRef]

23. Herz, J.; Siffrin, V.; Hauser, A.E.; Brandt, A.U.; Leuenberger, T.; Radbruch, H.; Zipp, F.; Niesner, R.A. Expanding two-photon intravital microscopy to the infrared by means of optical parametric oscillator. Biophys. J. 2010, 98, 715-723. [CrossRef]

24. Chen, T.-S.; Zeng, S.-Q.; Luo, Q.-M.; Zhang, Z.-H.; Zhou, W. High-order photobleaching of green fluorescent protein inside live cells in two-photon excitation microscopy. Biochem. Biophys. Res. Commun. 2002, 291, 1272-1275. [CrossRef] [PubMed]

25. Drummond, D.R.; Carter, N.; Cross, R.A. Multiphoton versus confocal high resolution z-sectioning of enhanced green fluorescent microtubules: Increased multiphoton photobleaching within the focal plane can be compensated using a Pockels cell and dual widefield detectors. J. Microsc. 2002, 206, 161-169. [CrossRef]

26. Kalies, S.; Kuetemeyer, K.; Heisterkamp, A. Mechanisms of high-order photobleaching and its relationship to intracellular ablation. Biomed. Opt. Express 2011, 2, 805-816. [CrossRef] [PubMed]

27. Graham, D.J.L.; Tseng, S.-F.; Hsieh, J.-T.; Chen, D.J.; Alexandrakis, G. Dependence of Two-Photon eGFP Bleaching on Femtosecond Pulse Spectral Amplitude and Phase. J. Fluoresc. 2015, 25, 1775-1785. [CrossRef] [PubMed]

28. Drobizhev, M.; Stoltzfus, C.; Topol, I.; Collins, J.; Wicks, G.; Mikhaylov, A.; Barnett, L.; Hughes, T.E.; Rebane, A. Multiphoton photochemistry of red fluorescent proteins in solution and live cells. J. Phys. Chem. B 2014, 118, 9167-9179. [CrossRef] [PubMed]

29. Ji, N.; Magee, J.C.; Betzig, E. High-speed, low-photodamage nonlinear imaging using passive pulse splitters. Nat. Methods 2008, 5, 197-202. [CrossRef] [PubMed]

30. Donnert, G.; Eggeling, C.; Hell, S.W. Major signal increase in fluorescence microscopy through dark-state relaxation. Nat. Methods 2007, 4, 81-86. [CrossRef]

31. Kawano, H.; Nabekawa, Y.; Suda, A.; Oishi, Y.; Mizuno, H.; Miyawaki, A.; Midorikawa, K. Attenuation of photobleaching in two-photon excitation fluorescence from green fluorescent protein with shaped excitation pulses. Biochem. Biophys. Res. Commun. 2003, 311, 592-596. [CrossRef]

32. Field, J.J.; Carriles, R.; Sheetz, K.E.; Chandler, E.V.; Hoover, E.E.; Tillo, S.E.; Hughes, T.E.; Sylvester, A.W.; Kleinfeld, D.; Squier, J.A. Optimizing the fluorescent yield in two-photon laser scanning microscopy with dispersion compensation. Opt. Express 2010, 18, 13661-13672. [CrossRef]

33. Xi, P.; Andegeko, Y.; Weisel, L.R.; Lozovoy, V.V.; Dantus, M. Greater signal, increased depth, and less photobleaching in two-photon microscopy with 10fs pulses. Opt. Commun. 2008, 281, 1841-1849. [CrossRef]

34. Wang, L.; Jackson, W.C.; Steinbach, P.A.; Tsien, R.Y. Evolution of new nonantibody proteins via iterative somatic hypermutation. Proc. Natl. Acad. Sci. USA 2004, 101, 16745-16749. [CrossRef]

35. Molina, R.S.; Qian, Y.; Wu, J.; Shen, Y.; Campbell, R.E.; Drobizhev, M.; Hughes, T.E. Understanding the Fluorescence Change in Red Genetically Encoded Calcium Ion Indicators. Biophys. J. 2019, 116, 1873-1886. [CrossRef]

36. Drobizhev, M.; Hughes, T.E.; Stepanenko, Y.; Wnuk, P.; O’Donnell, K.; Scott, J.N.; Callis, P.R.; Mikhaylov, A.; Dokken, L.; Rebane, A. Primary role of the chromophore bond length alternation in reversible photoconversion of red fluorescence proteins. Sci. Rep. 2012, 2, 688. [CrossRef] [PubMed]

37. Habuchi, S.; Cotlet, M.; Gensch, T.; Bednarz, T.; Haber-Pohlmeier, S.; Rozenski, J.; Dirix, G.; Michiels, J.; Vanderleyden, J.; Heberle, J.; et al. Evidence for the isomerization and decarboxylation in the photoconversion of the red fluorescent protein DsRed. J. Am. Chem. Soc. 2005, 127, 8977-8984. [CrossRef]

38. Shu, X.; Shaner, N.C.; Yarbrough, C.A.; Tsien, R.Y.; Remington, S.J. Novel chromophores and buried charges control color in mFruits. Biochemistry 2006, 45, 9639-9647. [CrossRef] [PubMed]

39. Ghosh, D.; Acharya, A.; Tiwari, S.C.; Krylov, A.I. Toward understanding the redox properties of model chromophores from the green fluorescent protein family: An interplay between conjugation, resonance stabilization, and solvent effects. J. Phys. Chem. $B$ 2012, 116, 12398-12405. [CrossRef] [PubMed]

40. Fuenzalida Werner, J.P.; Huang, Y.; Mishra, K.; Janowski, R.; Vetschera, P.; Heichler, C.; Chmyrov, A.; Neufert, C.; Niessing, D.; Ntziachristos, V.; et al. Challenging a Preconception: Optoacoustic Spectrum Differs from the Optical Absorption Spectrum of Proteins and Dyes for Molecular Imaging. Anal. Chem. 2020, 92, 10717-10724. [CrossRef]

41. Molina, R.S.; King, J.; Franklin, J.; Clack, N.; McRaven, C.; Goncharov, V.; Flickinger, D.; Svoboda, K.; Drobizhev, M.; Hughes, T.E. High throughput instrument to screen fluorescent proteins under two-photon excitation. Biomed. Opt. Express 2020, 11, 7192-7203. [CrossRef]

42. Guild, J.B.; Xu, C.; Webb, W.W. Measurement of group delay dispersion of high numerical aperture objective lenses using two-photon excited fluorescence. Appl. Opt. 1997, 36, 397-401. [CrossRef]

43. Dickson, L.D. Characteristics of a propagating gaussian beam. Appl. Opt. 1970, 9, 1854-1861. [CrossRef]

44. Drobizhev, M.; Molina, R.S.; Hughes, T.E. Characterizing the Two-photon Absorption Properties of Fluorescent Molecules in the 680-1300 nm Spectral Range. Bio-Protocol 2020, 10, e3498. Available online: https:/ / www.ncbi.nlm.nih.gov/pmc/articles/PMC7 409827/ (accessed on 23 December 2021). [CrossRef]

45. Xu, C.; Webb, W.W. Multiphoton Excitation of Molecular Fluorophores and Nonlinera Laser Microscopy. In Topics in Fluorescence Spectroscopy, Vol. 5, Nonlinear and Two-Photon Induced Fluorescence; Lakowicz, J.R., Ed.; Plenum Press: New York, NY, USA; London, UK, 1997; Chapter 11; pp. 471-540. 